\title{
Urheberrecht
}

\author{
Rainer Kuhlen*
}

\section{UrhWissG - das neue Wissenschaftsurheberrecht bleibt regulierungstechnisch überspezifisch problematisch}

https://doi.org/10.1515/iwp-2017-0048

Zusammenfassung: Das am 30. Juni 2017 im Bundestag beschlossene sogenannte Urheberrechts-Wissensgesellschafts-Gesetz (UrhWissG) wird aus der Perspektive von Bildung und Wissenschaft diskutiert. UrhWissG ist eine Reaktion auf die offensichtlichen Probleme mit den bestehenden, auf Bildung und Wissenschaft bezogenen Bestimmungen des Urheberrechts. Auf diese Probleme wird in Abschnitt 2 eingegangen. Die vorgesehenen neuen Schrankenregelungen werden im Detail vorgestellt (Abschnitt 3) und kritisch (ablehnend und konstruktiv weiterführend) diskutiert (Abschnitt 4). UrhWissG wird zu einigen Verbesserung für die Nutzung publizierten Wissens führen und allgemein für Rechtssicherheit sorgen. Der Vorrang von Schrankenregelungen gegenüber Lizenzangeboten der Verlagswirtschaft und die Pauschalvergütung für die schrankenbedingte Nutzung von Werken bzw. Teilen von ihnen sind im Gesetz festgeschrieben. Dennoch bleibt die von der Koalition an sich versprochene Allgemeine Bildungs- und Wissenschaftsschranke (ABWS) in Form einer generellen Klausel weiter eine Herausforderung für die politische Regulierung. Offenbar war die Zeit für eine umfassendere Reform des Wissenschaftsurheberrechts noch nicht gekommen. (Abschnitt 5). In der abschließenden Gesamtbewertung (Abschnitt 6) wird davor gewarnt, das nun beschlossene Wissenschaftsurheberrecht erst einmal als befriedet einschätzen, so dass es auf längere Zeit nicht mehr auf die politische Agenda kommen müssen. Es bleibt abzuwarten, ob durch die (derzeit weiter noch intensiv diskutierte) Vorgabe einer Urheberrechts-Richtlinie der EU

\footnotetext{
Anmerkung: Der vorliegende Beitrag, der zwar auch auf die Entwicklung im Gesetzgebungsverfahren, aber in erster Linie auf die am 30. Juni 2017 im Bundestag beschlossene Endversion des UrhWissG eingeht, ersetzt den Online-Artikel (DOI: https://doi.org/10.1515/iwp2017-0027). Alle angegebenen URL wurden am 12. Juli 2017 überprüft.

*Kontaktperson: Prof. em. Dr. Rainer Kuhlen, Bogotastraße 4, 14163

Berlin, E-Mail: rainer.kuhlen@uni-konstanz.de.

http://orcid.org/0000-0002-4497-6422
}

ein Umsetzungszwang in den EU-Mitgliedsländern entsteht, dem sich auch Deutschland nicht entziehen kann. Der Fokus richtet sich jetzt auf die EU. Im Anhang (Abschnitt 7 und 8) werden die Texte der acht neuen Schrankenregelungen sowie die Änderungen mit Blick auf die Nationalbibliothek aufgeführt.

Deskriptoren: Bibliothek, Bildung, UrhWissG, EuGH, Kopie, Lizenz, Gesetzgebung, Urheberrecht, Vergütung, Verlag, Wissenschaft und Technik, BGH, Deutschland

\section{Copyright reform in Germany - more legal certainty, but not really future-oriented}

Abstract: The recent reform of the German copyright law will be discussed from the perspective of education and science. This new law (abbreviated in German „UrhWissG) promises overcome the deficits of the existing copyright exceptions and limitations as far as education and science are concerned. We discuss these deficits in Sect. 3. The new exceptions will be explained in detail in Sect. 3 and their pros and cons will be discussed in Sect. 4. In its coalition treaty of 2013, the German government promised to introduce a general copyright clause in favour of education and science (GCC). In Chap. 5 we evaluate whether the new UrhWissG keeps this promise or at least, whether the new paragraphs can be considered a step in the right direction towards a modern science copyright. Compared to the old exceptions, there are some considerable improvements and UrhWissG creates some legal certainty, but there are still too many restrictions to the free use of published knowledge for the purpose of research and education. In general, the new law is not really future-oriented and takes only half-heartedly the potentials of modern information and communication technologies into account. The objective of a GCC should be still on the political agenda. (Sect. 6) The eight new copyright exceptions are listed in the appendix (Sec. 7). 
Descriptors: Copyright, General Copyright Clause, Education, Exceptions and Limitations, Germany, Libraries, $\mathrm{Pu}-$ blishing Companies, Remuneration, Research

\section{Urheberrechts- Wissensgesell- schafts-Gesetz - UrhWissG}

\subsection{Stadien bis zum Gesetz}

Anfang Februar 2017 hatte das Bundesministerium der Justiz und für Verbraucherschutz (BMJV) einen Referentenentwurf (RefE) für ein Gesetz zur Angleichung des Urheberrechts an die aktuellen Erfordernisse der Wissensgesellschaft (UrhWissG) vorgelegt. ${ }^{1}$ Nach vielen Jahren, in denen die Weiterentwicklung des Wissenschaftsurheberrechts eher durch die Rechtsprechung (der Gerichte, vor allem durch den Bundesgerichtshof) als durch die Rechtsetzung (der politischen Instanzen) vorgenommen wurde, versuchte jetzt die Politik, das Heft des Handelns wieder selbst in die Hand zu nehmen.

Das BMJV fasste seinen Entwurf wie folgt zusammen. ${ }^{2}$

Der Vorschlag:

- „regelt die künftigen Nutzungsbefugnisse für Unterricht, Forschung und Wissensinstitutionen möglichst konkret;

- verzichtet so weit wie möglich auf unbestimmte Rechtsbegriffe;

- weitet die Nutzungsbefugnisse aus, soweit unionsrechtlich zulässig und fachlich geboten;

- koppelt die erlaubten Nutzungen i.d.R. an einen gesetzlichen Anspruch der Urheber auf angemessene Vergütung, der über Verwertungsgesellschaften geltend zu machen ist;

- enthält erstmals eine urheberrechtliche Regelung zum „Text and Data Mining“;

- regelt unter anderem die „Anschlusskopie“ bei der Nutzung von Terminals;

- bereinigt die vorhandenen Schranken-Vorschriften und

- fügt erstmals eine plausible Binnenstruktur in den hochkomplexen Abschnitt 6 des Urheberrechtsgesetzes zu den Schrankenbestimmungen ein.“

1 Urheberrechts-Wissensgesellschafts-Gesetz - UrhWissG - http:// www.bmjv.de/SharedDocs/Gesetzgebungsverfahren/Dokumente/Ref E_UrhWissG.pdf?_blob=publicationFile\&v $=1$.

2 http://www.bmjv.de/SharedDocs/Gesetzgebungsverfahren/DE/Urh WissG.html.
Zudem wurde von den meisten Reaktionen auf den Entwurf der Vorteil der dadurch erzielten Rechtssicherheit betont. Ob Rechtssicherheit auch mit Zukunftssicherheit verträglich sein kann - auf diese entscheidende Frage wollen wir am Ende eine Antwort versuchen.

Das BMJV hat mit der Veröffentlichung seines Vorschlags zugleich die davon betroffenen Kreise aufgefordert, zu dem Entwurf Stellung zu nehmen. Die bis zum 27. Februar 2017 eingegangenen (93) Stellungnahmen wurden erfreulicher Weise (und bislang in der Politik nicht immer üblich) öffentlich zugänglich gemacht (unter der URL in Fußnote 3).

An die Stelle der bisherigen primär auf Bildung und Wissenschaft bezogenen Schrankenregelungen (vor allem die $\S \S 52 \mathrm{a}, 52 \mathrm{~b}$ und Teile von 53 sowie 53 a) wird eine ganze Serie von neuen Regelungen treten. Das BMJV verwendet dafür zum ersten Mal anstatt der bisherigen Bezeichnung „Schranken“ die Formulierung „Gesetzlich erlaubte Nutzungen für Unterricht, Wissenschaft und Institutionen“. Das ist zu begrüßen - wird dadurch sehr deutlich der gesetzlich verbindliche Charakter von solchen Bestimmungen betont und damit die Priorität von „Schrankenregelungen“ gegenüber vertraglichen Lizenzregelungen. Schranken erlauben (in einem festzulegenden Umfang) die genehmigungsfreien Nutzungen urheberrechtsgeschützter Werke. Sie sind keine bloße Ausnahmen, sondern als Begrenzung der exklusiven Rechte der Rechteinhaber (Urheber und Verwerter) ein Recht im öffentlichen Interesse.

Das Bundeskabinett hat am 12. April 2017 diesen Gesetzentwurf für eine Reform des Urheberrechts beraten und mit Änderungen beschlossen (aus RefE wurde RegE). Der Bundesrat hat am 12. Mai 2017 Empfehlungen dazu abgegeben $^{3}$, auf die hier im Folgenden aber nicht mehr eingegangen wird, weil keiner der Änderungsvorschläge des Bundesrats in der Antwort der Bundesregierung berücksichtigt wurde.

Der RegE wurde ohne Änderungen in der ersten Lesung im Bundestag am 19. Mai 2017 verabschiedet und dann im Rechtsausschuss über eine öffentliche Anhörung am 29. Juni 2017 diskutiert ${ }^{4}$ und mit einer Empfehlung an die Bundesregierung für die 2. und 3. Lesung zurückgegeben. Durch den Rechtsausschuss wurden gegenüber dem RefE, aber auch gegenüber dem RegE weitere

3 https://www.bundesrat.de/SharedDocs/drucksachen/2017/0301-0 400/312-17(B).pdf?_blob=publicationFile\&v=1.

4 Die Stellungnahme der Experten sind dokumentiert unter: https:// www.bundestag.de/ausschuesse18/a06/anhoerungen/stellungnahm en/508522; die des Verfassers auch unter: http://www.kuhlen.name/ MATERIALIEN/Publikationen2017/RK-Text-Rechtsausschuss-240520 17.pdf. 
Änderungen bzw. Einschränkungen der Nutzung vorgenommen. Und kurz vor der Abstimmung verständigten sich die Fraktionen der in der Regierung vertretenen Parteien nach intensivem Lobbying der Presse (initiiert durch FAZ $^{5}$ ) noch auf eine weitere Einschränkung, nämlich Zeitungsartikel von den Schrankenbestimmungen ( $\S 50 \mathrm{a}$, b, c, e, f) und vom Web-Harvesting durch die Nationalbibliothek auszunehmen. Am 30. Juni wurde das UrhWissG im Bundestag in 2. und 3. Lesung beschlossen, gegen die Stimmen der Linke und mit Enthaltungen der Grünen. Beide Parteien hatten bis zum RegE das Vorhaben mitgetragen, wenn auch mit vielen kritischen Einwänden. Mit den letzten Änderungen war für sie eine rote Linie überschritten.

Der Bundesrat hat am 8. Juli 2017 das Gesetz passieren lassen, ohne den Vermittlungsausschuss anzurufen (zustimmungspflichtig sind Urheberrechtsgesetze für den Bundesrat nicht - Urheberrecht ist Bundessache). Ein letzter Versuch, das Gesetz noch zu ändern, erschien wohl als aussichtslos und hätte das Gesetz als Ganzes möglicherweise gefährdet. Das konnte auch nicht im Interesse der Länder liegen.

Das Gesetz kann damit zum 1. März 2018 in Kraft treten. Sozusagen in letzter Minute wurde auch beschlossen, die Geltung der neuen Paragraphen auf fünf Jahre erst einmal zu begrenzen. ${ }^{6}$ Nach vier Jahren soll eine Evaluierung der Auswirkungen der Reform stattfinden.

\subsection{Struktur von UrhWissG}

Insgesamt werden in dem Unterabschnitt 4 von UrhWissG acht neue Paragraphen 60 a-60 h eingeführt. Dies wird als der Kern der Reform bezeichnet. (die Texte der acht Paragraphen im Anhang unter Abschnitt 7)

Die anderen Unterabschnitte von UrhWissG (1-3 und 5-6) behandeln andere gesetzlich erlaubte Nutzungen (Unterabschnitt 1); Regelungen zur Vergütung (Unterabschnitt 2); Weitere gesetzlich erlaubte Nutzungen (Unterabschnitt 3); Besondere gesetzlich erlaubte Nutzungen verwaister Werke (Unterabschnitt 5) und Gemeinsame Vorschriften für gesetzlich erlaubte Nutzungen (Unterabschnitt 6).

5 Vgl. dazu die Pressemitteilung des Aktionsbündnisses Urheberrecht für Bildung und Wissenschaft: http://www.urheberrechtsbuend nis.de/pressemitteilung0517.html.de.

6 Die fünf Jahre sollen nach der Erläuterung im Schlussantrag für das UrhWissG auch dafür genutzt werden, eine zentrale Online-Lizenzierungsplattform aufzubauen (Bundestagsdruck 18/13014, S. 5 - http:// dip21.bundestag.de/dip21/btd/18/130/1813014.pdf).
Dazu wird in Artikel 2 eine „Änderung des Gesetzes über die Deutsche Nationalbibliothek" vorgenommen und in Artikel 3 eine Änderung des Patentgesetzes.

Zusätzlich einschlägig für Bildung und Wissenschaft ist in Unterabschnitt 1 die Erweiterung des Zitatrechts ( $\$ 51$ UrhG) über einen neuen Satz 3, durch den die Zitaterlaubnis auch für „eine Abbildung und sonstige Vervielfältigungen der zitierten Werke“ gegeben wird. Das ist für die Praxis der Nutzung hilfreich.

Ansonsten konzentriert sich die Darstellung hier auf den erwähnten Kern mit den $\S \S 60 \mathrm{a}-60 \mathrm{~h}^{7}{ }^{7}$

In Abschnitt 3 werden die einzelnen Bestimmungen der acht neuen Normen im Detail besprochen. In Abschnitt 4 werden diese Bestimmungen kritisch überprüft. Die Vorschläge im RefE (also im Entwurf des BMJV) wurden von den meisten Organisationen und Personen aus Bildung und Wissenschaft als akzeptabel angesehen und im öffentlichen Diskurs unterstützt, vor allem deshalb, weil eine Pauschalierung der Vergütung für schrankenbedingte Nutzung und der Vorrang von Schrankenregelungen gegenüber Lizenzangeboten der Verlage im Reformvorschlag festgeschrieben wurde.

Allerdings wurde die Unterstützung nur sozusagen zähneknirschend gegeben, da das eigentliche Ziel, nämlich eine Allgemeine Bildungs- und Wissenschaftsschranke (ABWS) in das Urheberrecht einzuführen, nach vielen Jahren der Diskussion und nach dem Versprechen im Koalitionsvertrag von 2013 nicht erreicht wurde. Zwar wurde von der Politik die Verabschiedung der Reform als ABWS gefeiert und das Ziel eines wissenschaftsfreundlichen zeitgemäßen Urheberrechts als erreicht bezeichnet, aber das trifft tatsächlich nicht zu. In Abschnitt 5 wird daher noch einmal auf die Ziele und Notwendigkeit einer ABWS eingegangen.

Die Unterstützung des Reformvorhabens bröckelte nach den verschiedenen Stadien (s. oben) bis zur endgültigen Verabschiedung erheblich ab, und das Zähneknirschen nahm zu. $\mathrm{Zu}$ viele Zugeständnisse hatten dann wieder die Verwertungsorganisationen der Wissenschaftsverlage und die Pressemedien der Politik abringen können. Allerdings entschloss sich niemand aus Bildung und Wissenschaft zu einer öffentlichen Ablehnung des Vorhabens. Als zu wichtig wurden die beiden erhalten gebliebenen Punkte angesehen: Pauschalierung der Vergütung und Schrankenvorrang - allerdings wurde dieser Vorrang

7 Eine erste Stellungnahme des Autors zum Vorschlag des BMJV liegt auf den Websites von iuwis und von iRights vor http://www.iuwis.de/ blog/refe-urhgwissg-\%E2 \%80 \%93-keine-abws-daf $\%$ C3 \%BCr-8-kern spaltungen-respekt-aber-respekt-ist-nicht-alles; https://irights.info/ artikel/referentenentwurf-urheberecht-wissensgesellschaft-bildungswissenschaftsschranke/28355. 
am Ende in $\S 60 \mathrm{~g}$ etwas abgeschwächt. Für die Oppositionsparteien im Bundestag war das Ergebnis am Ende nicht mehr akzeptabel.

\section{Die Vorgeschichte des Wissenschaftsurheberrechts}

Das UrhWissG ist zweifellos eine Reaktion auf die offensichtlichen Probleme mit den bestehenden, auf Bildung und Wissenschaft bezogenen Bestimmungen des Urheberrechts.

Vor allem die beiden Reformen des Urheberrechts 2003 und 2008 (Erster und Zweiter Korb), durch die in der Umsetzung der Vorgaben der InfoSoc-Richtlinie der EU aus dem Jahr 2001 ein den digitalen Entwicklungen angemessenes wissenschaftsfreundliches Urheberrecht entstehen sollte, haben sich nicht als ausreichend erwiesen. Die Gründe dafür sind zahlreich, z. B.

- Die verschiedentlich verwendeten unbestimmten Rechtsbegriffe wie „veröffentlichte kleine Teile eines Werkes, Werke geringen Umfangs sowie einzelne Beiträge aus Zeitungen oder Zeitschriften“ (in § 52a) haben große Rechtsunsicherheit bewirkt, so dass zu vielen Regelungen, vor allem zu denen in den $\S \S 52 \mathrm{a}$ und $52 \mathrm{~b}$, Klagen der Verleger gegen nach ihrer Meinung zu weitgehende Nutzungsangebote der Bibliotheken geführt wurden: Diese Verfahren erstreckten sich i.d.R. über viele Jahre, bis sie dann vom Bundesgerichtshof (BGH) und zum Teil sogar erst durch den Europäischen Gerichtshof (EuGH) entschieden bzw. dann an die zuständigen Landes- oder Oberlandesgerichte zurück überwiesen wurden.

- Geklagt wurde von Verlagen (letztlich erfolglos) auch gegen Folgehandlungen wie Speichern und Ausdrucken von digitalen Materialien, die von den Bibliotheken entsprechend $\S 52 \mathrm{~b}$ bereitgestellt wurden.

- Als unglücklich erwiesen sich auch vage Formulierungen wie „Veranschaulichung im Unterricht“, wobei das „im“ von Kritikern des § 52a wortwörtlich genommen wurde, bis dann endlich vor den Gerichten geklärt wurde, dass eigentlich „des“ Unterrichts gemeint sei, weil sonst die gesamte Regelung keinen Sinn mache. Seitdem ist klar, dass auch Vor- und Nachbereitung des Unterrichts sowie Prüfungsvorbereitungen durch $\S 52$ a mit gedeckt sind.

- Viele Regelungen haben sich angesichts des technologischen Standes als unsinnig erwiesen, wie z.B. dass für den Kopienversand für Bibliotheken nur grafische Dateien (Faksimile) verschickt werden dürfen (ent- sprechend §53a), also keine voll-digitalen Dateien, welche die Weiterverarbeitung erlauben würden. Oder die Vorschrift, dass digitalisierte Objekte aus dem Bestand der jeweiligen Bibliothek nur an den Leseplätzen (!) in den Räumen der Bibliothek eingesehen werden dürfen (entsprechend $\S 52 \mathrm{~b}$ ), obgleich heute jeder berechtigte Nutzer über die Netzwerke von Orten seiner Wahl (z. B. über VPN) Zugriff haben könnte bzw. sollte.

- Unklar blieb, ob und inwieweit vertragliche Lizenzangebote Priorität gegenüber den Nutzungserlaubnissen in den Schrankenregelungen haben sollen.

- Unklar ist auch geblieben, in welcher Form (pauschal oder individuell) und in welcher Höhe die in den Normen vorgesehenen Vergütungen geregelt werden sollen. ${ }^{8}$

Alle diese Einschränkungen der Schrankenregelungen, die aus heutiger Sicht in ihrer Kleinlichkeit und Unzeitgemäßheit eher satireverdächtig wirken, sind in erster Linie der Sorge der Verleger bzw. dem intensiven und weitgehend erfolgreichen Lobbying des sie repräsentierenden Börsenvereins des deutschen Buchhandels geschuldet. Sie wollten sich gegen die ihrer Ansicht nach zu intensive, genehmigungsfreie Nutzung der von ihnen auf den Markt gebrachten Werke wehren. Wenn es nach dem Börsenverein ginge, wären Schrankenregelungen überhaupt überflüssig, da der freie Markt mit Lizenzierungsvereinbarungen dies alles besser regeln könnte. Alle diese Argumente, Behauptungen und leider auch Polemiken kehrten von dieser Seite jetzt in der Diskussion um das UrhWissG wieder. $^{9}$

Verbesserungen der Regelungen von 2003 und 2008 über einen Dritten Korb mit dem Ziel eines wissenschaftsfreundlichen Urheberrechts wurden von der Großen Koalition ab 2009 angekündigt. Dazu ist es aber nie gekommen. Es hat keine umfassende Lösung für das angestrebte Ziel

8 Die Kontroverse um pauschale Vergütung oder einzelfallbezogene Nutzung hatte Ende 2016 zu einem heftigen Konflikt an den Hochschulen geführt, der vorübergehend durch die Rücknahme des zwischen KMK und VG-Wort abgeschlossenen Rahmenvertrags ausgesetzt wurde. Dieser Rahmenvertrag hatte eine Individualvergütung vorgesehen, die von den Hochschulen als unzumutbar zurückgewiesen wurde. Bis Ende September 2017 soll von KMK und VG-Wort, jetzt ergänzt um HRK, ein neuer Vorschlag vorgelegt werden. Durch den Vorschlag des UrhWissG, eine Pauschalvergütung vorzusehen, werden die KMK, VG Wort und HRK kaum umhin kommen, diese auch in die neue Regelung ab 1. Oktober 2017 aufzunehmen.

9 Vgl. die Website: https://www.publikationsfreiheit.de/; vgl. dagegen: http://www.urheberrechtsbuendnis.de/pressemitteilung0217. html.de. 
gegeben, sondern lediglich ganz am Ende der damaligen Legislaturperiode einen Nachschlag mit der Einführung eines Zweitverwertungsrechts (nach einer kommerziellen Erstpublikation) über $\S 38,4$ und einer neuen Schrankenregelung für verwaiste und vergriffene Werke. Allerdings gab es auch hier wieder schwer nachvollziehbare Einschränkungen. So wurde das Zweitverwertungsrecht für solche Werke verweigert, welche aus der grundfinanzierten Hochschulforschung entstanden sind. Nur Werke aus der Drittmittelforschung oder der außeruniversitären Forschung wurden begünstigt - mit der Begründung, dass daran ein höheres öffentliches Interesse bestünde.

Wie oben erwähnt, sind einige der Ungereimtheiten in den Schrankenregelungen durch Gerichtsurteile auf höchster Ebene beseitigt worden. Trotzdem besteht bezüglich der Anwendung der durch die letzten Reformen eingebrachten Schrankenregelungen weiter große Rechtsunsicherheit: Die alten Schranken sind nach wie vor in ihren ursprünglichen Formulierungen im Gesetz. Es ist einem Nutzer kaum zuzumuten, sich durch die Interpretationen der verschiedenen Gerichtsentscheidungen durchzuarbeiten, um dadurch in einigen Punkten Rechtssicherheit zu gewinnen. Deshalb ist es zu begrüßen, dass das BMJV die Initiative der Rechtssetzung wieder ergriffen hat, die strittigen Paragraphen streichen lassen will und ganz neue Paragraphen mit größerer Rechtssicherheit einführen will. Das BMJV fasst selber die Defizite der bisherigen Regelungen in seiner Problembeschreibung wie folgt zusammen (Referenz s. Fußnote 2):

1. Das Urheberrechtsgesetz (UrhG) enthält in den $\S \S 44 \mathrm{aff}$. UrhG [gemeint sind die Schrankenregelungen - RK] derzeit eine Vielzahl kleinteiliger, an unterschiedlichen Stellen geregelter gesetzlicher Erlaubnistatbestände zugunsten von Unterricht und Wissenschaft. Sie sind für die Adressaten - also Schülerinnen und Schüler, Lehrerinnen und Lehrer, Studierende und Lehrende an Hochschulen, Mitarbeiterinnen und Mitarbeiter von Bibliotheken, Archiven etc. schwierig aufzufinden und anzuwenden: Sie enthalten zudem etliche auslegungsbedürftige Begriffe, die Gegenstand gerichtlicher Auseinandersetzungen waren oder noch immer sind.

2. Zugleich haben Digitalisierung und Vernetzung die Möglichkeiten der Schaffung, Verbreitung und die Nutzung urheberrechtlich geschützter Inhalte verändert. Diesen Veränderungen werden die bestehenden Schrankenbestimmungen für Wissenschaft und Unterricht, die sich teilweise an „analogen“ Nutzungen orientieren, nicht mehr vollständig gerecht: Zeitgemäßer Gebrauch, der das Potenzial der modernen Wissensgesellschaft ausschöpfen würde, unterbleibt daher teilweise oder aber er geschieht rechtswidrig zugleich zum Nachteil auch der Rechtsinhaber, die weder bei der unterbliebenen noch bei einer rechtswidrigen Nutzung eine Vergütung erhalten.

\section{Die Schrankenregelungen im Vorschlag des BMJV im Einzelnen}

\section{$3.1 \S 60$ a Unterricht und Lehre}

In den ersten beiden Absätzen von $\S 60$ a wird geregelt, welche urheberrechtsrelevante Nutzung in welchem Umfang für wen und für welchen Zweck privilegiert ist.

Es wird nun auch im Gesetz bzw. in der Erläuterung dazu klargestellt, was unter Veranschaulichung des Unterrichts (nicht mehr „im“) bedeutet: „Die Veranschaulichung kann „im“ Unterricht erfolgen, aber auch davor oder danach. Daher erfasst die Vorschrift zum einen auch die Vor- und Nachbereitung der eigentlichen Unterrichtsstunden und zum anderen auch die Prüfungsaufgaben und Prüfungsleistungen, die im Verlauf und zum Abschluss des Unterrichts erstellt werden, sowie die Vor- und Nachbereitung von Prüfungen.“

Auch der Fernunterricht wird explizit begünstigt. Vorgenommene Vervielfältigungen in dem vorgesehenen Umfang dürfen für die öffentliche Wiedergabe und öffentliche Zugänglichmachung genutzt werden.

Klargestellt wird auch, dass Begünstigte nicht nur die Lehrenden und Prüfer, sondern auch die Teilnehmer an der jeweiligen Veranstaltung sind. Das ist im Sinne des didaktisch gewünschten selbstbestimmten Lernens zu begrüßen. Allerdings dürfen die „Schüler“ (ist wohl Oberbegriff für alle Lernenden) die bereitgestellten Materialien nicht an Teilnehmer nachfolgender Jahrgänge weitergeben.

Ein weiterer Versuch wird unternommen abzuklären, was „unter nicht-kommerziell“ zu verstehen ist. Dafür sei nicht der institutionelle Bezug ausschlaggebend (also auch Privatschulen werden durch $\S 60$ a begünstigt), sondern allein, ob der Unterricht darauf ausgerichtet ist, „Gewinn zu erzielen“ oder nicht. Ob diese Klärung in der Praxis ausreicht, muss sich erst noch zeigen,

Auf die Verwendung unbestimmter Rechtsbegriffe wie „kleine Teile“ wird verzichtet. Von größeren Werken dürfen bis zu 15 Prozent genutzt (vervielfältigt, verbreitet und öffentlich zugänglich gemacht werden) (im RefE waren 25 Prozent vorgesehen und auch vom Bundesrat gefordert). Für die Nutzung von Abbildungen, Beiträgen aus Fachzeitschriften sowie für „sonstige Werke geringen Umfangs“ 
gibt es keine Umfangsbeschränkung. Interpretieren wir dies einmal so, dass damit auch Beiträge in Konferenzproceedings und anderen Sammelbänden gemeint sind. In letzter Minute wurden nach intensivem Lobbying der Zeitungsverleger Artikel aus Zeitungen von der Privilegierung in $\S 60 \mathrm{a}$ - wie auch in den $\S \S 60 \mathrm{~b}$, c, e und $\mathrm{f}$ - ausgenommen, obgleich die genehmigungsfreie Nutzung von Zeitungsartikeln für Zwecke von Forschung und Lehre/Lernen schon seit 2003 durch $\S 52$ a erlaubt war - ohne erkennbaren Nachteil für die Pressemedien.

Schwierig ist weiter eindeutig festzulegen, was mit „öffentlich“ gemeint ist. Sicher nicht, wie man umgangssprachlich vermuten würde, die allgemeine Öffentlichkeit. Das UrhG bestimmt „öffentlich“ in § 12 (regelt Veröffentlichung) und $\S 15$ (regelt öffentliche Nutzung) unterschiedlich. Das UrhWissG lehnt sich an die Definition von § 15 an. Danach „handelt es sich bereits um Öffentlichkeit, wenn nur wenige nicht miteinander verbundene Personen das Werk wahrnehmen können. “10 Oder anders: öffentlich gilt, „solange die Nutzer eines Werkes nicht durch eine persönliche Beziehung miteinander verbunden sind" "11. Daher ist die Nutzung durch Lehrende, Prüfer und Lernende als öffentliche Zugänglichmachung erlaubt - allerdings nur für diese Gruppen, nicht für die Allgemeinheit bzw. die allgemeine Öffentlichkeit. Allerdings werden in der Erläuterung zu § 60 a Nutzungen für „Schulklassen und andere kleine, regelmäßig zusammen unterrichtete Gruppen“ (überraschend) als nicht öffentlich angesehen, so dass diese Nutzungshandlungen nicht als ,urheberrechtlich relevant" angesehen werden. Lehrer dürfen daher z. B. Filme in der Gänze zeigen, aber nicht den Film in der Gänze vervielfältigen. Vervielfältigungen (gemeint ist Kopieren) dürfen nur bis zu 15 Prozent des Films gemacht werden, und entsprechend dürfen von dem Film dann auch nur 15 Prozent in der Schulklasse gezeigt werden. Auch dürfen Filme, die im Prinzip ja jetzt unter den Bedingungen der Absätze 1 und 2 genutzt werden dürfen, nicht aus einer Vorführung im Kino oder anderen Live-Veranstaltungen „mitgeschnitten oder live gestreamt werden.“

Eine strikte Bereichsausnahme gibt es (wie auch schon in dem jetzigen $\S 52 \mathrm{a}$ ) für Werke, die ,ausschließlich für den Unterricht an Schulen geeignet, bestimmt und entsprechend gekennzeichnet" sind (Abs. 3, 3). Hierdurch soll der Primärmarkt für Schulbuchverlage weiter gesichert werden. Daher müsste jeder Lehrende bzw. seine Instituti-

$10 \mathrm{https} / / /$ www.bundestag.de/blob/406648/fd9a3c33f848b086d53b 70d6be053a48/wd-10-044-14-pdf-data.pdf.

11 https://boehmanwaltskanzlei.de/kompetenzen/medienrecht/urh eberrecht/urheberrechtsschutz/voraussetzungen/verwertungsrechte/ 678-der-begriff-der-oeffentlichkeit-im-urhebergesetz. on beim jeweiligen Rechtsinhaber (oft sind es mehrere) um Erlaubnis nachsuchen. Da dies in der Praxis nicht realistisch ist, einigten sich die Länder und VG-Wort in der Vergangenheit auf einen Gesamtvertrag ${ }^{12}$ für erlaubte Nutzungen in Schulen in dem Umfang, wie es $\S 52$ a vorsieht (also „kleine Teile“ etc.). Es ist davon auszugehen, dass dadurch, dass $\S 60$ a jetzt Gesetz wird, ein Gesamtvertrag die dort vorgesehenen Nutzungsbedingungen übernehmen wird.

Von Verlegerseite wurde die Forderung gestellt, dass es auch eine Bereichsausnahme für die Nutzung von Lehrbüchern an Hochschulen geben müsse. Das ist in $\S 60 \mathrm{a}$ nicht vorgesehen, da die Nutzungssituation dort grundsätzlich eine andere sei als im föderalen System an Schulen.

\section{$3.2 \S 60 \mathrm{~b}$ Unterrichts- und Lehrmedien}

$\S 60 \mathrm{~b}$ Unterrichts- und Lehrmedien ist eine eher ungewöhnliche, bislang im UrhG nicht vorgesehene Schrankenregelung. Es besteht für das BMJV offenbar ein öffentliches Interesse daran, Hersteller von Unterrichts- und Lehrmedien dergestalt zu begünstigen, dass sie in ihre Produkte „zur Veranschaulichung des Unterrichts und der Lehre an Bildungseinrichtungen ( $\$ 60 \mathrm{a}$ ) zu nicht-kommerziellen Zwecken“ Teile von urheberrechtsgeschützten Werken genehmigungsfrei in ihre Sammlungen einbinden dürfen. Mit Sammlungen sind Werke gemeint, „die Werke einer größeren Anzahl von Urhebern vereinigen“. Von größeren Werken dürfen nur Teile von bis zu zehn Prozent genommen werden; Werke kleineren Umfangs dürfen, wie in $\S 60$ a vorgesehen, in der Gänze dafür genutzt werden (aber keine Zeitungsartikel). Abweichend von anderen Schrankenregelungen dürfen Vergütungen nicht pauschal, sondern müssen genau nach der aktuellen Verwendung abgerechnet werden.

\section{$3.3 \S 60 \mathrm{c}$ Wissenschaftliche Forschung}

$\S 60 \mathrm{c}$ Wissenschaftliche Forschung (zusammen mit § $60 \mathrm{a}$ ) ist als Folgeregelung für den dadurch zu streichenden $\S 52 \mathrm{a}$ UrhG anzusehen. Klargestellt wird, dass sich jedermann, der wissenschaftliche Forschung betreibt, auf diese

12 http://www.bibliotheksverband.de/fileadmin/user_upload/DBV/ vereinbarungen/2014_02_27_GV_52aurhg_nutzungen_schulen.pdf. Für die Zeit von August 2013 bis Juli 2017 zahlten die Länder an die VG-Wort pauschal Euro 2.240.000. 
Regelung berufen kann, also auch „unabhängige Forscher“, „Privatgelehrte“.

Anders als $\S 52 \mathrm{a}$ UrhG verzichtet $\S 60 \mathrm{c}$ auf unbestimmte Rechtsbegriffe. Auch die von $\S 52$ a übernommene Formulierung in Abs. 1, 1 ,bestimmt abgegrenzten Kreis von Personen für deren eigene wissenschaftliche Forschung“ dürfte durch die Erläuterung geklärt sein: „Unerheblich ist, ob alle Personen an derselben Einrichtung tätig sind. Auch innerhalb loser Forschungsverbünde dürfen Materialien genutzt werden.“

Für die nach $\S 60$ c, Abs. 1 erlaubten Nutzungshandlungen der Vervielfältigung, Verbreitung und öffentliche Zugänglichmachung dürfen nun bis zu 15 Prozent (ursprünglich waren 25 Prozent vorgesehen) eines Werke genehmigungsfrei, aber vergütungspflichtig genutzt werden.

Abbildungen, einzelne Beiträge aus derselben Fachzeitschrift oder wissenschaftlichen Zeitschrift (aber nicht Artikel aus Zeitungen), sonstige Werke geringen Umfangs und vergriffene Werke dürfen in der Gänze genutzt werden (vermutlich auch Werke aus Proceedings und anderen Sammelbänden, obgleich dies nicht explizit erwähnt wird; aber die Formulierung „sonstige Werke geringen Umfangs" liegt dies nahe).

Die mediale Gestalt der Werke ist nicht auf Text beschränkt. Die Nutzungserlaubnisse von $\S 60 \mathrm{c}$, Abs. 1 gelten demnach jetzt auch (so wird es in der Begründung festgestellt) für „Filmwerke und grafische Aufzeichnungen von Werken der Musik“ „um die Erforschung solcher Werke zu erleichtern. Eine Beeinträchtigung der normalen Verwertung solcher Werke ist dadurch nicht zu befürchten.“ Die alten Bereichsausnahmen aus $\S 52$ a somit fallen fort. Eine Bereichsausnahme ist allerdings geblieben: Die Aufnahmen von Live-Veranstaltungen vor Ort und eine spätere öffentliche Zugänglichmachung sind verboten. Irritierend ist hier, dass unter das Verbot auch öffentliche Vorträge fallen sollen. Sind damit auch Vorträge von Wissenschaftlern z. B. auf Fachkonferenzen gemeint? Das machte keinen Sinn.

\section{$3.4 \S 60 \mathrm{~d}$ Text und Data Mining}

Die neu aufgenommene „Vorschrift [zum TDM] ermöglicht es, auf gesetzlicher Grundlage Werke mit Inhalten aller Art automatisiert auszuwerten, z. B. Werke mit Texten, Daten, Bildern, Tönen oder audiovisuellen Inhalten, um damit nicht-kommerzielle wissenschaftliche Forschung zu betreiben." (Erläuterung) TDM selber ist auch nach herrschender Meinung (so auch im UrhWissG vertreten) nicht urheberrechtlich relevant, wohl aber sind es die vorbereitenden Handlungen zur Herstellung der Korpora aus oft heterogenen Quellen (vor allem die Vervielfältigung/das
Kopieren, aber auch Formatanpassungen und Anbringen zusätzlicher Metainformationen), die bei den meisten TDM-Anwendungen anfallen. Mit bloßen Streaming ist es i.d.R. nicht getan. Auf die Nutzung kann sich, wie schon in $\S 60 \mathrm{c}$ vorgesehen, jedermann berufen, der diese Art (nicht-kommerzieller) Forschung betreiben will.

Die Nutzungshandlungen für Vervielfältigungen und öffentliche Zugänglichmachung werden privilegiert, nicht die für Verbreitung. Die öffentliche Zugänglichmachung ist zum einen auf Nutzer im Umfang wie in $\S 60 \mathrm{c}$ (,,bestimmt abgegrenzten Kreis von Personen“) beschränkt sowie auf einzelne Dritte, welche die erzielten Ergebnisse für anfallende Reviews (Überprüfungen/Bewertungen) anhand der für das TDM zusammengestellten und aufbereiteten Korpora nutzen wollen. Nur die Korpora dürfen von diesen Reviewern verwendet werden, nicht die Ausgangs-/ Rohmaterialien.

TDM-Anwender dürfen dies nur zu nicht-kommerziellen Zwecken (wie in $\S \S 60 \mathrm{a}$ und 60c definiert) nutzen. Entscheidend ist nicht der institutionelle Bezug, sondern nur der Zweck. Zu beachten ist, dass die TDM-Erlaubnis nicht den Zugang zu den geschützten Materialien schafft. Dieser Zugang muss über die Bestände in der/n eigenen Einrichtung/en (i.d.R. die Bibliothek/en) oder auch über Fernleihe erfolgen. Dies trifft im Übrigen auch für die anderen Schrankenregelungen zu. Schrankenregelungen sind kein Freibrief zur „Piraterie“ oder zur „Enteignung“ von privatem Eigentum.

Zum Schutz der Interessen der (kommerziellen) Rechtsinhaber müssen nach Beendigung der TDM-Arbeiten die Ausgangsmaterialien und die Korpora bei den TDM-Anwendern gelöscht und damit auch die Zugänglichmachung beendet werden. Einrichtungen wie Bibliotheken, Archive und Museen (definiert in den $\S \S 69$ e und 60 f) dürfen jedoch die im TDM-Prozess zusammengestellten Ausgangsmaterialien und die aufbereiteten Korpora archivierend speichern. Im Schrankentext ist nicht die Rede davon, dass diese gespeicherten Materialien zur Überprüfung wieder eingesehen werden dürfen. Die Formulierung in der Erläuterung legt aber nahe, dass dies möglich bzw. sogar erforderlich ist: „Die Forscher wollen und müssen die für ihre Forschung benutzten Inhalte weiterhin in Gänze verfügbar halten, um die Zitierbarkeit, Referenzierbarkeit und die Überprüfung der Einhaltung wissenschaftlicher Standards zu ermöglichen.“

\section{$3.5 \S 60$ e Bibliotheken}

Diese Schrankenregelung begünstigt, wie die Regelungen in den anderen $\S \S$ auch, nur Handlungen von Bibliothe- 
ken, die nicht kommerziellen Zwecken dienen. Bislang war nicht ausdrücklich legitimiert, dass als Voraussetzung z. B. für „Zwecke der Zugänglichmachung, Indexierung, Katalogisierung, Erhaltung und Restaurierung“ Vervielfältigungen der urheberrechtsgeschützten Materialien erstellt werden dürfen. Das ist jetzt, wie schon durch Gerichtsentscheidungen für richtig befunden, geregelt. (Abs. 1) Diese Vervielfältigungen dürfen auch an andere Bibliotheken für Zwecke der Restaurierung weitergegeben werden. (Abs. 2) An andere Bibliotheken dürfen ,restaurierte Werke sowie Vervielfältigungsstücke von Zeitungen, vergriffenen oder zerstörten Werken aus ihrem Bestand" verliehen werden. Auch die Verbreitung solcher Werke in „öffentlicher Ausstellung oder zur Dokumentation des Bestandes der Bibliothek" ist erlaubt. (Abs. 3)

Die für die Nutzung entscheidende öffentliche $\mathrm{Zu}$ gänglichmachung von digitalisierten Werken dürfen Bibliotheken aus ihrem Bestand weiterhin (so wie jetzt in $\S 52$ b UrhG geregelt) nur „an Terminals in ihren Räumen“ für Nutzer zu Forschungszwecken oder privaten Studien ermöglichen (Abs. 4). Von größeren Werken darf jeder Nutzer pro Sitzung (einmal am Tag?) zehn Prozent für den eigenen Gebrauch vervielfältigen, d.h. z. B. auf einen Stick speichern oder die zehn Prozent ausdrucken. ${ }^{13}$ Annexhandlungen sind auch schon vom Bundesgerichtshof für den alten $\S 52 \mathrm{~b}$ als erlaubt angesehen worden, sind aber jetzt auch durch das Gesetz legalisiert.

Die Zehn-Prozent-Beschränkung gilt auch für den Versand auf Bestellung für nicht-kommerzielle Zwecke (Abs. 5). Für kleinere Werke, Zeitschriftenartikel etc. gelten keine Beschränkungen. Artikel aus Zeitungen sind ausgeklammert (entsprechend den Regelungen in $\S 60$ a und c). Der Versand ist jetzt (anders als noch in $\S 53 \mathrm{a}$ ) ,technologieneutral und ohne Vorrang von Verlagsangeboten“ (Erläuterung). Die alte Beschränkung, dass Artikel nur als grafische Datei bzw. per Fax versandt werden dürfen, ist somit weggefallen. Weggefallen ist aber auch, das Bibliotheken nicht mehr an Benutzer in kommerziellen Einrichtungen liefern dürfen. (Abs. 5)

Alle diese Bestimmungen, bis auf die für den Versand, gelten auch für $\S 60 \mathrm{f}$ Archive, Museen und Bildungseinrichtungen.

\section{$3.6 \S 60 \mathrm{~g}$ Gesetzlich erlaubte Nutzung und vertragliche Nutzungsbefugnis}

Im ersten Absatz wird der gesetzlichen Regelung in den Schrankenbestimmungen Priorität gegenüber vertraglichen Regelungen (Lizenzen) eingeräumt. Nach Absatz 2 gilt diese Priorität jedoch nicht für die in $\S 60$ e Bibliotheken mögliche öffentliche Zugänglichmachung von digitalisierten Werken aus dem Bestand der jeweiligen Bibliotheken bzw. für die unter $\S 60 \mathrm{f}$ angeführten Institutionen (Archive etc.).

\section{$3.7 \S 60 \mathrm{~h}$ Angemessene Vergütung der gesetzlich erlaubten Nutzungen}

Für alle Nutzungen in den Regelungen im Unterabschnitt 4 des UrhWissG (also § 60a-60 f) haben die Rechtsinhaber einen Anspruch auf Vergütung. Begründet wird dies dadurch, das der Gesetzgeber grundsätzlich verpflichtet sei, den vermögenswerten Gehalt des Urheberrechts dem Urheber bzw. nach Übertragung der Verwertungsrechte als Nutzungsrechte an einen Verleger auch diesem zuzuordnen. (Abs. 1) Ausgenommen von der Vergütungspflicht ist die „öffentliche Wiedergabe für Angehörige von Bildungseinrichtungen und deren Familien“, nicht jedoch für diese die öffentliche Zugänglichmachung. (Abs. 2)

Für die Erhebung der Nutzung und der Berechnung der angemessenen Vergütung werden Pauschalierungsverfahren bzw. repräsentative Stichproben für genügend gehalten. Aus der Erläuterung kann man ableiten, dass wegen des großen Aufwandes für individualisierte Erhebung und Abrechnung Bibliotheken bzw. die ihnen zugeordneten Einrichtungen davon absehen würden, von den Schrankenregelungen Gebrauch zu machen. ${ }^{14}$ Eine solcher Verzicht würde, ,jedoch die Gemeinwohlinteressen beeinträchtigen“, die sich u.a. ,auf das Ziel guter und umfassender Bildung“ richten. (Erläuterung zum UrhWissG) Die Pauschalvergütung gilt nicht für Nutzungen nach $\S 60 \mathrm{~b}$.

14 Dies zeichnete sich ja auch schon Ende letzten Jahres bei der Weigerung der Hochschulen ab, dem Rahmenvertrag für die Nutzung nach § 52a UrhG zwischen KMK und VG-Wort beizutreten, der Individualerhebung und -abrechnung vorsah. Diese Weigerung hätte zur Folge gehabt, dass z. B. ein Großteil der digitalen Semesterapparate nicht mehr hätte genutzt werden können.
13 Gegen diese Regelung richtet sich besonders stark die Verlagsindustrie, da nach zehn jeweils für sich erlaubten Nutzungen das ganze Werk kopiert werden könnte. 


\section{Reaktionen auf das UrhWissG-Vorhaben}

\subsection{Kritik aus der Sicht der Verlagswirtschaft (Wissenschafts- und Presseverlage)}

Enteignungsvorwurf: Wissenschaftsverlage und der Pressemedien sahen nicht nur den Schutz des Urhebers und die Vertragsfreiheit im Rahmen der Privatautonomie durch die Reform stark eingeschränkt, sondern befürchteten als Folge des neuen Gesetzes eine „faktische Enteignung“ der Verlage (vor allem der Börsenverein ${ }^{15}$ ). Auch einzelne Autoren aus der Wissenschaft wandten sich vehement gegen das neue Gesetz (exemplarisch hierfür der Artikel von Roland Reuss mit der Überschrift „Der Staat legalisiert die Enteignung von Urhebern“, geschrieben nach der Verabschiedung des Gesetzes). ${ }^{16}$

Unvereinbar mit dem Dreistufentest: Die Ausweitung der Nutzungsbedingungen, z.B. die Erhöhung der Anteile an den zu nutzenden Werken von bislang 12 Prozent auf jetzt 15 Prozent (ursprünglich vorgesehen 25 Prozent) sei kaum noch mit dem Dreistufentest vereinbar (vor allem nicht mit Stufe 2, die die normale Verwertung sichern soll) und bedrohe den Primärmarkt der Verlage. ${ }^{17}$

Keine Pauschalvergütung: Die in $\S 60 \mathrm{~h}$ präferierte Pauschalabrechnung wird kritisiert (vor allem von Verlagen und Verwertungsgesellschaften, vgl. URL über Fußnote 3); entweder sollte grundsätzlich nach jeder einzelnen Nutzung abgerechnet werden (bevorzugt) oder es sollten angemessene Kompromissmodelle zwischen pauschaler und individueller Vergütung entwickelt werden.

Kein Vorrang von Schrankenregelungen: Der Vorrang von Schrankenregelungen gegenüber Lizenzangeboten/-vereinbarungen wurde kritisiert. Diese ruiniere die Primärmärkte für alle Informationsprodukte.

Bereichsausnahme für Lehrbücher gefordert: Vor allem sei eine Bereichsausnahme für Lehrbücher, nicht nur für Schulbücher, erforderlich. (Börsenverein, vgl. URL über Fußnote 3)

15 https://www.boersenblatt.net/artikel-boersenverein_zum_geplan ten_urheberrechts-wissensgesellschafts-gesetz.1319338.html.

16 https://www.nzz.ch/feuilleton/urheberrechtsreform-in-deutschla nd-der-staat-legalisiert-die-enteignung-von-urhebern-ld.1304125; vgl. auch die Verlagsinitiative https://www.publikationsfreiheit.de/. 17 Der Dreistufentest gilt sozusagen als Lackmus-Test für die rechtliche Gültigkeit von Schrankenregelungen im Urheberrecht. Sie sind, in der Formulierung des WIPO Copyright Treaty (1996), Art 10, nur erlaubt ,in certain special cases that do not conflict with a normal exploitation of the work and do not unreasonably prejudice the legitimate interests of the author." [Hervorhebung R.K.].
Tenor: Von der Verlagswirtschaft wurde das Vorhaben in der Gänze abgelehnt, und es wurde mit einer Klage vor dem Bundesverfassungsgericht gedroht.

\subsection{Grundsätzliche Zustimmung aus der Wissenschaft}

Eine große Mehrheit der beim BMJV eingegangenen Stellungnahmen schätzte den ursprünglichen RefE des BMJV als grundsätzlich positiv ein. ${ }^{18}$ Den Tenor gibt der Titel einer Pressemitteilung des Aktionsbündnisses Urheberrecht für Bildung und Wissenschaft an, dessen Sprecher der Verfasser dieses Beitrags ist: „Der Weg ist noch nicht zu Ende - aber die Richtung stimmt“"19. Von den meisten zustimmenden Stellungnahmen wurde anerkannt, dass das vom BMJV zentral verfolgte Ziel der Rechtssicherheit als erreicht anzusehen sei.

Wie schon zu Beginn angedeutet, sind die $\S \S 60 \mathrm{a}-60 \mathrm{~h}$ zwar nicht als eine Allgemeine Bildungs- und Wissenschaftsschranke anzusehen ist (dazu vgl. Kapitel 5), sie wurden aber von der Fachwelt als wichtiger Schritt in Richtung eines bildungs- und wissenschaftsfreundlichen Urheberrechts bzw. eines allgemeinen Wissenschaftsurheberrechts angesehen. Viele der Regelungen in den Paragraphen $60 \mathrm{a}$ bis $60 \mathrm{~h}$ sind, wie in Abschnitt 3 im Detail beschrieben, sinnvoll, überfällig und für Nutzer besser verständlich und sind wohl auch praxisnah und in den Interessen sorgfältig abgewogen. Die ursprünglich vorgesehene Erhöhung der Nutzungserlaubnis wurden von vielen Stellungnahmen als angemessen bezeichnet, die jetzt beschlossene auf 15 Prozent eher nur als marginaler Fortschritt angesehen.

Fazit: Die Regelungen in den neuen Paragraphen wurden in vielen Details, speziell wegen der Festschreibung des rechtlichen Vorrangs gegenüber Lizenzangeboten oder -vereinbarungen des Marktes, als Fortschritt gegenüber den alten Regelungen aus dem Zweiten und Dritten Korb der Urheberrechtsreformen ab 2003 angesehen. Auch mit der Präferenz für eine Pauschalabrechnung der schrankenbezogenen Nutzungen kommt das UrhWissG den in For-

18 So z.B. (trotz zahlreicher Änderungsvorschläge) grundsätzlich GRUR; KMK, dbv; Allianz der Wissenschaftsorganisationen; Forschungsgruppe Ethik des Kopierens am Zentrum für interdisziplinäre Forschung (ZiF) in Bielefeld; dbv; DHd - Digital Humanities im deutschsprachigen Raum; DINI; Wikimedia Deutschland; IDS; zahlreiche Universitäten Forschungs- und Wissenschaftsorganisationen. (alles über die URL in Fußnote 3).

$19 \mathrm{http} / /$ www.urheberrechtsbuendnis.de/pressemitteilung0117.ht ml.de. 
schung und Lehre Arbeitenden, nicht zuletzt den Auszubildenden, entgegen. Die empirischen Daten aus der einschlägigen Osnabrücker Studie und auch die heftig ablehnenden Reaktionen auf den Rahmenvertrag zu § 52 Ende 2016, der eine Einzelabrechnung vorsah, zeigen deutlich, dass eine solche individualisierte Erhebung und Abrechnung keine Akzeptanz in Forschung und Lehre finden kann.

\subsection{Kritik, Defizite, Verbesserungsvorschläge}

Die vor allem zu Beginn des Verfahrens im Prinzip positive Resonanz aus der Sicht der Wissenschaft kann aber nicht darüber hinwegtäuschen, dass es im Verlauf des Verfahrens (vgl. Unterabschnitt 1.1) zum einen einige Rücknahmen der freieren Nutzungsmöglichkeiten von publiziertem Wissen gab und dass zum andern vielen weitergehenden Forderungen im UrhWissG nicht entsprochen wurde ganz zu schweigen davon, dass die Ziele der an sich versprochenen ABWS, entgegen der Einschätzung der Regierungsvertreter in der 2. und 3. Lesung im Bundestag (Maas, Flisek, Schieder, Wanka, Kretschmer) ${ }^{20}$, nicht erreicht wurden (vgl. Abschnitt 5).

1. Es ist nicht mehr zeitgemäß, dass weiterhin nicht von außerhalb auf die digitalisierten Bestände der Bibliotheken zugegriffen werden kann. Benutzer müssen nach wie vor in die Bibliotheksräume gehen, haben keinen legitimen elektronischen Zugriff (z.B. über VPN) von wo auch immer. Für Bibliotheken sind die Räume im elektronischen Umfeld aber nicht länger nur physische, sondern auch virtuelle Räume. Auch der aktuelle Vorschlag der EU-Kommission hält die Beschränkung auf eine exklusive Nutzung in den physischen Räumen der Bibliotheken nicht mehr für zeitgemäß.

2. Bei der Definition von Bildungseinrichtungen, die durch $\S 60$ a privilegiert werden, hätten auch Volkshochschulen oder MOOCs (Massive Open Online Courses) explizit mit berücksichtigt werden sollen.

3. Wichtige Beiträge wie Konferenzbeiträge und Artikel in Sammelbänden jeder Art sollten explizit (zumindest in den Erläuterungen) in die Nutzungserlaubnisse, z.B. in den $\$ \S 60 \mathrm{a}$ und $60 \mathrm{c}$ UrhWissG, einbezogen werden. Solche Beiträge sind in vielen Disziplinen wichtiger als Aufsätze in Zeitschriften. Die Formulie-

20 http://dipbt.bundestag.de/doc/btp/18/18244.pdf; Zusatztagesordnungspunkt 13 . rung „sonstige Werke geringen Umfangs“ ist vielleicht nicht ausreichend.

4. Die Text-and Data-Mining (TDM)-Schranke ( $§ 60 \mathrm{~d})$ ist sicher auch für den Gesetzgeber nicht das letzte Wort. Sie sollte auch nicht strikt auf nicht-kommerzielle Nutzung beschränkt werden (auch wenn es sinnvoll ist, das Kriterium der Nicht-Kommerzialität nicht institutionell zu begründen). Die gerade bei TDM wichtigen Kooperationen mit der IT-Wirtschaft werden so behindert oder sogar unmöglich gemacht. Fraglich auch, warum rein kommerzielle Nutzungen nicht auch privilegiert werden sollen, wenn dafür angemessene Vergütungen gezahlt werden. So werden kommerzielle Anbieter gezwungen, sich auf aufwändige Lizenzverhandlungen mit kommerziellen Anbietern einzulassen.

5. TDM-Nutzungen sollten nicht vergütungspflichtig sein, da der Schaden für die Rechtsinhaber durch TDM-Nutzung minimal ist. So sieht es auch die entsprechende Passage (Erwägungsgrund EG 13) des Vorschlags der EU-Kommission für TDM vor.

6. Die in Bibliotheken (nach TDM-Auswertungen) rechtlich erlaubt gespeicherten (TDM)-Korpora müssen für die Überprüfung der Ergebnisse bei Bedarf wieder neu zugänglich gemacht werden können (wie gesagt, nicht für neue Nutzungen, sondern eben nur zur Überprüfung). Dies ist aus wissenschaftsethischen Gründen unverzichtbar.

7. Durch eine Erweiterung der Nutzungen nach $\S 60$ e, Abs. 3 wäre es sinnvoll gewesen, den Bibliotheken zur Bewerbung und Anzeige ihres Bestandes, die Vervielfältigungen nicht nur zu verbreiten, sondern auch öffentlich zugänglich machen. Hätte auch für Archive etc. in $\S 60 \mathrm{f}$ gelten sollen.

8. Leider ist $\S 60$ e, Abs. 5 so restriktiv gehalten worden, dass der Versand auf Bestellung für Einrichtungen mit kommerziellen Nutzungszwecken nicht erlaubt ist. Eine solche (eng begrenzte) Nutzung durch die Wirtschaft läge sicherlich im öffentlichen Interesse.

9. Für Bibliotheken ist bislang keine Regelung vorgesehen, dass sie E-Books sowohl erwerben als auch ausleihen können. Das Argument, dass die Überlegungen dazu noch nicht abgeschlossen seien, vermag nicht $\mathrm{zu}$ überzeugen. Praktikable, auch den Interessen der Verlage entgegenkommende Vorschläge von Seiten der Bibliotheken liegen vor. ${ }^{21}$

21 Vgl. http://www.bibliotheksverband.de/dbv/themen/e-books-inbibliotheken.html; vgl. auch den entsprechenden Antrag der LINKE, der aber nicht vom Bundestag angenommen wurde: http://dip21.bun destag.de/dip21/btd/18/054/1805405.pdf. 
10. Leider ist darauf verzichtet worden, eine Öffnungsklausel im UrhWissG vorzusehen. Dies ist von de la Durantaye (mit Verweis auf Schack; Schack-Text unter Fußnote 34) bei der Anhörung im Rechtsausschuss gefordert worden..$^{22}$ Öffnungsklauseln sind ein Mittel im Urheberrecht, die Schrankenbestimmungen zwar möglichst eng zu halten, aber für bestimmte Nutzungen, vielleicht auch für solche, die zum Zeitpunkt der Gesetzgebung noch unbekannt waren, Ausnahmen für weitergehendere Nutzungen zu erlauben. Als Beispiel wäre zu nennen, dass für Zwecke des Reviewing auch ganze Werke genehmigungsfrei (vielleicht dann sogar vergütungsfrei) genutzt werden könnten und nicht nur die generell erlaubten 15 Prozent des Werkes.

11. Auf eine Quantifizierung der Nutzungshandlungen (derzeit variierend zwischen 10, 25 und 75 Prozent) wäre besser ganz verzichtet worden. Entscheidend sollte allein der Zweck der Forschung bzw. der Ausbildungsaktivität sein: „Konsequenz wäre ein Verzicht auf eine Quantifizierung... Stattdessen könnte als Kriterium dienen, was in der Wissenschaft und in den Vorlesungen zur Veranschaulichung „benötigt“ wird bzw. was „erforderlich“ ist.“ (Stellungnahme hlb; URL über Fußnote 3) Dies ist nicht zuletzt auch der wichtigste Vorschlag in einer ABWS, die diesen Namen verdient. (vgl. Abschnitt 5)

12. Gänzlich unakzeptabel ist das Einknicken der Politik gegenüber dem Ansinnen der Presseverleger (initiiert durch ganzseitige Anzeigen der Herausgeber und Geschäftsführer der FAZ ${ }^{23}$ ), Zeitungsartikel aus der Privilegierung herauszunehmen. Diese Forderung war im gesamten Prozess nie ein Thema, zumal diese Erlaubnis schon seit 2003 durch $\S 52$ a (der jetzt abgeschafft wird) geregelt war, ohne dass dagegen je ein Protest von Seiten der Medien erhoben wurde oder negative Folgen dieser Regelung bekannt wurden. Forschung und Lehre dürfen somit nicht zuletzt die durchaus wissenschaftlich relevanten Artikel nicht mehr genehmigungsfrei nutzen, sondern müssen sich Lizenzen dafür bei den Verlagen besorgen.

13. Vollkommen überflüssig auch die zuletzt vorgenommenen Änderungen in $\S 16 \mathrm{a}$, Abs. 2, durch die auf Drängen der Presseverleger diesen deutlich ihr exklu-

22 http://www.bundestag.de/blob/508702/e212463a189075cb0126c c085a3fb22a/durantaye-data.pdf.

23 Vgl. dazu Eric W. Steinhauer - http://kapselschriften.blogspot. de/2017/05/die-urhberrechtsmarchen-der-faz-zum.html; vgl. Aktionsbündnis: http://www.urheberrechtsbuendnis.de/pressemitteilung05 17.html.de sives Recht auf die Vermarktung ihrer Archive zugesichert worden ist. Ein Eingriff in dieses Recht war über das neue Recht der Nationalbibliothek für das WebHarvesting ohnehin nie vorgesehen, da nur solche Texte „geerntet“ werden dürfen, die frei zugänglich sind bzw. solche nicht, für welche die langfristige Verfügbarkeit, z. B. durch Pressearchive, gesichert ist.

14. Im Rahmen der Neuordnung des Wissenschaftsurheberrechts hätten auch die Regelungen in den 95erParagraphen UrhG überprüft werden müssen. Dieser „alte Zopf“ aus der EU-Richtlinie von 2001, dem der Gesetzgeber in Deutschland 2003 meinte folgen zu müssen, ist nicht länger haltbar: Technische Schutzmaßnahmen (DRM) sollten in Bildung und Wissenschaft grundsätzlich nicht zum Einsatz kommen. Auch die Langzeitarchivierungsanstrengungen der Bibliotheken und Archive sollten nicht durch DRM behindert werden.

15. Die Gelegenheit der Reform des Wissenschaftsurheberrechts hätte auch dafür genutzt werden sollen, die 2014 gültig gewordenen Regelungen für ein Zweitverwertungsrecht in $\S 38$, Absatz 4 UrhG gerechter und praxisnäher zu gestalten. Die bislang ausgeschlossene grundfinanzierte Hochschulforschung müsste in das Zweitverwertungsrecht einbezogen werden. ${ }^{24}$ Dazu wäre nur eine leichte redaktionelle Änderung von $\S 38$, Absatz 4 erforderlich gewesen.

16. Ebenfalls ist die bei der letzten Urheberrechtsanpassung von 2014 beschlossene Vorschrift für die Nutzung von verwaisten Werken leider nicht korrigiert worden. Diese Vorschrift besagte, dass verwaiste Werke ${ }^{25}$ nur dann genutzt (vervielfältigt und öffentlich zugänglich gemacht) werden dürfen, wenn dem eine sorgfältige (höchst aufwändige) Suche (diligent search) vorausgegangen war. Die Bedingung einer solchen Suche erwies sich als Hindernis für die an sich erwünschte Massendigitalisierung für Zwecke digitaler Bibliotheken - auch wenn durch Anwendung der Regeln auf

24 Hierzu exemplarisch für die Allianz der Wissenschaftsorganisationen die Stellungnahme der Helmholtz Gemeinschaft: Zweitverwertungsrecht: Forschungsorganisationen kritisieren Einschränkungen. - https://www.helmholtz.de/wissenschaftspolitik/zweitverwert ungsrecht-forschungsorganisationen-kritisieren-einschraenkungen1560/; vgl. Rainer Kuhlen: Manifestierung der Dreiklassengesellschaft [zum Zweitverwertungsrecht]. Spektrum.de (Spektrum der Wissenschaft) 19.9.2013 - http://www.spektrum.de/kolumne/manifestier ung-der-dreiklassengesellschaft/1207757.

25 Verwaiste Werke sind Werke, die die zeitlichen Bedingungen für den urheberrechtlichen Schutz noch erfüllen und für die die Rechtsinhaber (Autor und/oder Verleger) nicht ausgemacht werden können. 
vergriffene Werke diese Restriktion teilweise umgangen werden konnte. ${ }^{26}$

17. Es wurde versäumt, die Regelungen für Vergütung für (meistens öffentlich finanzierte) Werke der Wissenschaft insgesamt und besonders für die Bereiche der Bildung noch einmal grundlegend in Frage zu stellen. Die EU-Kommission in ihrem aktuellen Vorschlag sieht z. B. für die Nutzungen im Bereich „cross-border education“ keine allgemeine Verpflichtung der Mitgliedsländer vor, und in Ländern in der EU wie in Estland, die auch auf die nach wie vor gültige InfoSocRichtlinie von 2001 verpflichtet sind, ist die Nutzung für Zwecke der Ausbildung genehmigungs- und vergütungsfrei. Möglich wäre es also.

18. Wie häufig könnten auch gut gemeinte Regelungen zu unerwünschten Nebenfolgen führen. So wurde schon in der Stellungnahme von GRUR zum RefE darauf hingewiesen ${ }^{27}$, dass die grundsätzliche Vergütungspflichtigkeit bei Schranken wie 60 a und 60 c dazu führen würde/könnte/müsste, dass die privilegierten Einrichtungen Nutzungsvergütungen zum einen auch für Werke zahlen müssen, die ihre eigenen Mitarbeiter erstellt haben, und zum andern auch für die nach Open Access an sich freigestellten Werke. (GRUR; vgl. URL über Fußnote 3) Das wäre so lange der Fall wie der „Entwurf nicht die Möglichkeit eines Verzichts auf Vergütungsansprüche für den Bereich der Open-Access-Dienste vorsieht." Von sich aus können Urheber nicht im Voraus auf Vergütungsansprüche verzichten..$^{28}$ Auch darauf hat der Gesetzgeber nicht reagiert.

19. Von Seiten des Aktionsbündnisses Urheberrecht für Bildung und Wissenschaft wurde als einzige Organisation die Vergütungspflichtigkeit für Werke, die in öffentlichen Umgebungen durch öffentlich finanzierte Wissenschaftler entstanden sind, gänzlich in Frage

26 Vergriffene Werke (definiert für Werke, die vor dem 1.1.1966 erschienen und die im Buchhandel vergriffen sind) dürfen von Bibliotheken, die diese Werke analog in ihren Beständen haben, im Rahmen von digitalen Bibliotheken für nicht-kommerzielle Zwecke über eine von der VG-Wort vergebene Nutzungslizenz digitalisiert und öffentlich zugänglich gemacht werden (geregelt u.a. durch den Rahmenvertrag zur Nutzung von vergriffenen Werken in Büchern http://www.bibliotheksverband.de/fileadmin/user_upload/DBV/ver einbarungen/2015_01_RV_vergriffene_Werke.pdf).

27 http://www.bmjv.de/SharedDocs/Gesetzgebungsverfahren/Stell ungnahmen/2017/Downloads/02102017_Stellungnahme_Grur_RefE_ UrhWissG.pdf?_blob=publicationFile\&v=1.

28 GRUR schlägt daher für $\S 60 \mathrm{~g}$ einen neuen Absatz 3 vor: „Eine Vereinbarung, durch welche der Urheber unentgeltlich ein einfaches Nutzungsrecht für jedermann einräumt, geht abweichend von Absatz 1 der gesetzlichen Erlaubnis vor. Dasselbe gilt, soweit der Urheber seinem Dienst- oder Arbeitgeber Rechte eingeräumt hat.“ gestellt. Diese Problematik kann hier nicht annähernd erschöpfend behandelt werden. Nur einige Hinweise:

a. Stärker als bislang sollte im Wissenschaftsurheberrecht berücksichtigt werden, dass in Bildung und Wissenschaft (anders als in der Belletristik oder in anderen Bereichen der Publikumsmärkte) das Interesse der Autorinnen und Autoren an einer Vergütung für die Publikation - bis auf wenige Ausnahmen der Textsorten und der Fächer i.d.R. sehr gering ist bzw. eine Vergütung i.d.R. nicht stattfindet. Oft genug muss für eine Publikation bzw. sogar schon für das Reviewing gezahlt werden.

b. Die entscheidende Währung in der Wissenschaft ist Reputation, nicht finanzielle Belohnung (obgleich natürlich Reputation oft genug auch positive finanzielle Konsequenzen hat).

c. Auf jeden Fall ist das Interesse jeden Autors an freier Nutzung (da er immer auch Nutznießer schon publizierter Werke ist) und dem damit verbundenen Reputationsgewinn ${ }^{29}$ höher einzuschätzen als das Interesse an einer Vergütung.

d. Im Normalfall der wissenschaftlichen Publikation wird weder eine Vergütung angeboten noch hat der Urheber wissenschaftlicher Texte Gelegenheit, seinen Verzicht auf Vergütung explizit zu artikulieren geschweige denn seinen Anspruch durchzusetzen. Verlage haben durchweg gegenüber den Urhebern die stärkere Verhandlungsposition. Zudem werden oft genug keine Verträge abgeschlossen. Der Verzicht auf Vergütung wird einfach als gegeben angenommen. Es kann daher im Normalfall keine Rede davon sein, dass ein wissenschaftlicher Urheber grundsätzlich frei über seinen Vergütungsanspruch verfügen kann.

e. Die Vergütungsfrage sollte auch in den Kontext der 2002 erfolgten Änderung des Gesetzes über Arbeitnehmererfindungen gestellt werden. Durch diese Änderung wurde das bis dahin bestehende Hoch-

29 "The incentive for academic authors to publish research results is mostly reputational rather than economic bestowing only indirect gains in the way of peer esteem and professional advancement. In fact, ever since the first scientific journals were founded (in the seventeenth century), publishers have rarely paid authors for their articles. This is also where the scientific publishing industry is distinguished from the traditional one, as the interests of commercial publishers and other information providers differ from those of scholarly authors, with the former usually pursuing a profit maximizing strategy. Furthermore, the vast majority of academic research is publicly funded." (Valentina Moscon: Academic Freedom, Copyright, and Access to Scholarly Works: A Comparative Perspective, p. 101). 
schullehrerpatentierungsprivileg abgeschafft, da aus Art 5, 3 GG nicht das Recht auf kommerzielle Nutzung (hier der Erfindung) abgeleitet werden könne. ${ }^{30}$ Wäre nicht der Analogieschluss von der ökonomisch sicher bedeutsamen „Erfindung“ auf in der Praxis ohnehin kaum vergütete „Publikationen"sinnvoll?

f. Ohnehin sind die meisten publizierenden Wissenschaftler über ihre Gehälter schon durch die Öffentlichkeit finanziert. Forschung und Publizieren gehört zu den Dienstverpflichtungen und müssen nicht zusätzlich monetär honoriert werden.

g. Entsprechend des von der Politik auch in Deutschland geforderten Open-Access-Publizierens wird dieses mehr und mehr zum Standard. Im Rahmen von Open-Access sollten ohnehin keine Vergütungsansprüche geltend gemacht werden.

In dieser Liste gibt es viele Konjunktive, viel „hätte“ - in der Tat: UrhWissG ist ein Schritt in die richtige Richtung, aber der Weg ist noch sehr weit bis zu einer an sich versprochenen ABWS bzw. zu einem wirklich wissenschaftsfreundlichen Urheberrecht.

\section{Die Forderung nach einer Allgemeinen Bildungs- und Wissenschaftsschranke (ABWS) besteht weiter}

Zugunsten einer ABWS hatte sich seit etwa fünf Jahren ein breiter politischer Konsens entwickelt. Auf die Forderung nach einer ABWS haben sich alle im Deutschen Bundestag vertretenen Parteienverständigt, ebenso der Bundesrat, die Enquete Kommission „Internet und Digitale Gesellschaft“, die Digitale Agenda 2014-2017 der Bundesregierung, die Allianz der Wissenschaftsorganisationen, die Kultusministerkonferenz und viele Organisationen aus der Zivilgesellschaft und der Wissenschaft ${ }^{31}$. Entsprechend hatte sich auch die jetzige Bundesregierung in ihrem Koalitionsvertrag von 2013 dieser Forderung angeschlossen. Auf S. 93 dieses Vertrags heißt es: „Wir werden den wichtigen Belangen von Wissenschaft, Forschung und Bildung stärker Rechnung tragen und eine Bildungs- und Wissenschaftsschranke einführen". In der Tat hat hier die Bundesregierung ein konkretes Versprechen gegeben. Es heißt: wir „werden“ und nicht wir „wollen“. ${ }^{32}$

Die Diskussion um die Einführung einer ABWS ist natürlich noch viel breiter. Die Pro- und Contra-Argumente werden durch die Beiträge in dem einschlägigen ZUMThemenheft $6 / 2016$ bereitgestellt. ${ }^{33}$

Die Forderung, in das Urheberrecht eine ABWS einzuführen, wurde im Wesentlichen durch zwei Argumente begründet:

(1) Die bisherigen, auf Bildung und Wissenschaft bezogenen Schrankenregelungen haben sich jede für sich und in ihrem Zusammenspiel als äußerst komplex, unzeitgemäß und für Bildung und Wissenschaft als eher behindernd als befördernd erwiesen. Eine punktuelle Verbesserung wurde kaum für realistisch angesehen. Daher sollten alle diese Schranken durch eine einheitliche ABWS ersetzt werden.

Dieses Ziel wurde durch UrhWissG erreicht.

(2) Es besteht die Gefahr, dass auch neue, auf konkrete Regulierungsanweisung setzende einzurichtende Schrankenregelungen schon sehr bald dem weiterhin zu erwartenden technologischen Wandels kaum gerecht werden können. Sind Schranken überspezifisch, veralten sie schnell. Wenn das Gesetz nicht ständig den Entwicklungen hinterherhinken soll und damit Rechtsunsicherheit erzeugt, sollte, so die Forderung, eine ABWS in das Urheberrechts-Gesetz eingeführt werden.

Eine ABWS mit einer entsprechenden Generalklausel könnte auf detaillierte Schrankenregelungen verzichten und sollte dem technologischen Wandel und den sich ändernden Verhaltensformen für den Umgang mit Wissen und Information, einschließlich innovativer Geschäftsmodelle der Verlagswelt, und weiterer Verbreitung von Open Modellen (Open Access, Open Educational Resources, Open Data) besser gerecht werden können.

32 Die Bundesregierung hat dies in ihrem Vorhaben zur „Digitalen Agenda“ bekräftigt: Insbesondere soll eine Bildungs- und Wissenschaftsschranke eingeführt werden“ (Digitale Agenda 2014-2017, S. 27).

33 ZUM 6/2016 mit den folgenden Autoren: de la Durantaye (mit einer Antwort auf Schack in ZUM 3/2016; vgl. FN 34); Michael Grünberger (Bayreuth); Guido F. Herrmann (Thieme-Verlag); Ole Jani (Berlin); Rainer Kuhlen; Sebastian Pech (München); Thomas Pflüger (MWFK-B-W); Robert Staats (VG-Wort); Eric W. Steinhauer (Hagen).
30 „Das Grundrecht des Artikels 5 Abs. 3 GG gebietet zwar nicht die Rechtsinhaberschaft des Hochschullehrer an seinen Forschungsergebnissen, denn die Forschungsfreiheit umfasst nicht das Recht auf kommerzielle Nutzung von Wissenschaft-Erfindungen“ (BT-Dr 14/5975 vom 9. Mai 2001).

31 Vgl. Rainer Kuhlen: Wie umfassend soll / darf / muss sie sein, die allgemeine Bildungs- und Wissenschaftsschranke? Zeitschrift für Geistiges Eigentum / Intellectual Property Journal, Volume 7, 2015 , Number 1, pp. 77-125. 
Für eine ABWS waren die Vorschläge aus den Oppositionsparteien am weitestgehende. Die Linke schlug vor (ganz im Sinne des Klausel-Charakters der ABWS), „die derzeit geltenden, kleinteiligen Ausnahmeregelungen in einer gemeinsamen Generalklausel für die Bereiche Bildung und Wissenschaft zusammenzufassen. Diese sollte sich nicht so sehr an einzelnen Nutzungsarten orientieren, sondern am Zweck. Erlaubt sollte sein, was zu Zwecken von Wissenschaft, Bildung und Forschung geschieht - im Gegensatz zum kommerziellen Bereich. ${ }^{\text {34 }}$ Sehr konkret formuliert es die Fraktion Bündnis 90/die Grünen in einem Antrag an den Bundestag vom 27. April 2016 (Drucksache $18 / 8245)^{35}$ :

Eine ABWS „würde es Lehrenden, Lernenden und Forschenden erleichtern, publizierte Werke jedweder medialer Art für den nicht gewerblichen, wissenschaftlichen Gebrauch grundsätzlich genehmigungsfrei und ohne Einschränkungen zu nutzen. Ziel muss es dabei sein, den für Bildung und Wissenschaft notwendigen Zugang zu digitalen Werken unter angemessenen und für alle Seiten fairen Bedingungen zu gewährleisten. Zugleich soll eine solche Regelung ermöglichen, die digitalen Potenziale für Bildung und Forschung in der Breite nutzen zu können." Und sie fordert die Bundesregierung direkt auf, „unverzüglich einen Gesetzentwurf für eine allgemeine Bildungs- und Wissenschaftsschranke im Urheberrecht vorzulegen."

Ich stelle im Folgenden die Ziele zusammen, die durch eine ABWS erreicht werden sollen. Ich verwende hierfür die Abkürzung ABWK, da es sich hierbei weniger um eine Schranke im herkömmlichen Sinne handeln sollte, sondern um eine (General)Klausel ${ }^{36}$. Der Vorteil einer solchen Klausel ist, dass sie nicht nur für Rechtssicherheit sorgt, sondern auch für Zukunftssicherheit. Sie ist so allgemein gehalten, dass nicht mehr für jede neue, bislang noch nicht bekannte Anwendung eine neue Schrankenregelung beschlossen werden muss (wie jetzt am Beispiel von TDM).

34 Vgl. https://www.linksfraktion.de/themen/a-z/detailansicht/wis senschaftsschranke/.

35 http://dip21.bundestag.de/dip21/btd/18/082/1808245.pdf; vgl. Tabea Rößner, Sprecherin für Medien, Kreativwirtschaft und Digitale Infrastruktur - http://tabea-roessner.de/category/uber-mich/

36 Was eine Allgemeine Bildungs- und Wissenschaftsklausel (ABWK) leisten soll. Zielvorstellungen, Prüfbausteine. November 2015 - Text unter http://www.urheberrechtsbuendnis.de/docs/Ziele-ABWK.pdf. Vgl. Rainer Kuhlen: Wie umfassend soll/darf/muss sie sein, die allgemeine Bildungs- und Wissenschaftsschranke? In: Zeitschrift für Geistiges Eigentum/Intellectual Property Journal, Volume 7, 2015, Number 1, pp. 77-125; Die Allgemeine Bildungs- und Wissenschaftsschranke im Urheberrecht nimmt Fahrt auf - Kreativität und Innovation werden die Gewinner sein. Information - Wissenschaft \& Praxis 2016; 67(1): 1-9.
1. Durch eine ABWK wird publiziertes Wissen für Zwecke von Bildung und Wissenschaft zu jeder Zeit, von jedem Ort und über ein beliebiges Medium genehmigungsfrei verfügbar sein.

2. Die ABWK unterstützt Kreativität von Forschung und Qualität von Ausbildung und fördert damit indirekt auch die Innovationsfähigkeit in der Wirtschaft.

3. Die ABWK ist im Einklang mit den Zielen der EU- und anderen völkerrechtlichen Vorgaben und befördert in einer offenen und zeitgemäßen Interpretation von Art. 5, 3, a der InfoSoc-Richtlinie die Weiterentwicklung der EU-Urheberrechtspolitik.

4. Die Regelungen in der ABWK haben Vorrang vor Vertragsvorgaben aus der Informationswirtschaft und können in Verträgen grundsätzlich nicht abgedungen werden.

5. Die Regelungen der ABWK für die Nutzung von publiziertem Wissen sind so festgelegt, dass jedermann Wissenschaftler, Lehrende und Lernende -verstehen kann, welche Nutzungen erlaubt sind.

6. Die ABWK ersetzt das bisherige komplizierte System der verschiedenen, Bildung und Wissenschaft betreffenden Schrankenregelungen.

7. Die ABWK privilegiert mit Blick auf Bildung die traditionellen und die elektronisch verteilt und kollaborativ organisierten Lehr- und Lernprozesse sowie die Formen selbstbestimmten und selbstorganisierten Lernens.

8. Die ABWK ist technikneutral und offen für technologische Weiterentwicklungen, wie z. B. aktuell durch Verfahren des Text und Data Mining. Neue Schrankenregelungen dafür wären nicht nötig. Entsprechende Tools dürfen frei zur Anwendung kommen bei Material, das für den Zugang bereits lizenziert wurde.

9. Für die ABWK ist, wenn dies überhaupt als erforderlich angesehen wird, ein Vergütungsverfahren vorgesehen, das durchweg auf pauschale Abrechnung abzielt. Einzelerfassung und -lizenzierung bzw. individuelle Abrechnung sind, nicht zuletzt wegen des hohen Aufwands für alle Betroffenen, nicht akzeptabel.

10. Das Vergütungsverfahren einer ABWK trägt der Besonderheit von Werken Rechnung, die durch öffentliche Finanzierung direkt oder indirekt unterstützt wurden. Diese Werke sind genehmigungs- und vergütungsfrei nutzbar.

11. Die ABWK regelt die Nutzungshandlungen von Informationsvermittlungseinrichtungen wie Bibliotheken, Archiven, Museen, in dem Umfang, wie er Zwecken von Forschung und Bildung dient.

12. Die ABWK unternimmt in der Zweckbestimmung der privilegierten Nutzung keine starke Abgrenzung zwi- 
schen kommerzieller und nicht kommerzieller Forschung.

Der umfassende Klausel-Charakter wird am besten durch Abs. 1 des Vorschlags des Aktionsbündnisses Urheberecht für Bildung und Wissenschaft getroffen:

\begin{abstract}
„Zulässig ist die Vervielfältigung und öffentliche Zugänglichmachung eines veröffentlichten Werkes für nicht kommerzielle Zwecke a) wissenschaftlicher Forschung für Mitglieder in formal eindeutig bestimmten Forschungsgruppen oder b) der Lehr- und Lernprozesse von Lehrveranstaltungen an Bildungseinrichtungen. Satz 1 gilt auch für Zwecke der Bestandserhaltung durch Einrichtungen wie öffentlich finanzierte Bibliotheken, Archive, Dokumentationen und Museen. Satz 1 gilt auch für die wissenschaftliche Forschung und Lehren und Lernen unterstützende Leistungen von in Satz 2 erwähnten Vermittlungsinstitutionen." ${ }^{37}$
\end{abstract}

Das UrhWissG hat bei der Regulierungsmethode der „eindeutigen Normsetzung“ gegenüber einer Generalklausel den Vorzug gegeben. Begründet wurde das damit, dass „auch Generalklauseln ... eine Konkretisierung [erfordern], um sie im Einzelfall handhabbar zu machen“. Verbindlich könnten diese Konkretisierungen und neue Nutzungsformen, so zeige es die Vergangenheit, oft nur durch langjährige gerichtliche Auseinandersetzungen geklärt werden.

Verwiesen wird bei der Ablehnung von Generalklauseln i.d.R. und so auch in der Erläuterung zum UrhWissG auf die „Fair use“-Klausel des 1. U.S.C. § 107 (US Copyright Act), welche, nicht zuletzt wenn sie bei neuen Nutzungen in Anspruch genommen wird, juristisch immer wieder neu überprüft werden muss. So geschehen bei der langjährigen juristischen Auseinandersetzung mit dem Google-BooksProjekt, welche letztlich als durch fair use gedecktes Vorhaben legitimiert wurde.

Das Argument, dass durch eine Generalklausel weiter Rechtsunsicherheit erzeugt wird, überzeugt nicht. Wie in dem obigen Vorschlag vorgeschlagen, reichte als Klausel die Rückbindung an den jeweiligen Zweck der wissenschaftlichen, nicht-kommerziellen Forschung und an die ebenfalls nicht auf kommerziellen Erwerb ausgerichtete Aus- und Fortbildung aus ${ }^{38}$ : Das ist rechtssicher eindeutig.

37 Vorschlag einer Allgemeinen Bildungs- und Wissenschaftsklausel -http://www.urheberrechtsbuendnis.de/abws-text.html.de 38 In seiner Stellungnahme zum RefE stellt der hlb dazu fest: „Gerechtfertigt kann die Nutzung nur dann sein, wenn sie benötigt wird und es hängt vom Einzelfall in der jeweiligen Lehrsituation und nicht von der gesetzgeberischen Entscheidung ab, ob das ganze Werk oder nur 25 Prozent eines Werkes benötigt wird“. (Stellungnahme über die URL in Fußnote 3).
Fazit: Es trifft wohl zu, dass die Zeit für eine vollständige Einlösung dieser Ziele noch nicht da ist. Dagegen scheinen, wohl auch für das BMJV, die Bundesregierung und die Mehrheit des Bundestags, einige unionsrechtliche internationale, vielleicht sogar verfassungsrechtliche Vorgaben zu sprechen.

Allein sind auch diese keine unabdingbaren, sozusagen in Stein gemeißelten Festschreibungen, sondern sie sind in ihrer zeitbedingten Interpretation offen. Wie schnell sich bisherige Rechtsetzung, Rechtsprechung und Interpretation des Grundgesetzes ändern können, zeigte sich bei dem am selben Tag wie das UrhWissG im Bundestag verabschiedete „Ehe für alle“-Gesetz.

Sollte man sich damit zufrieden geben, dass diese Ziele bzw. die umfassend angelegte ABWS nur als regulative Ideen zu verstehen seine, die die Handlungen und die festzulegenden Regulierungen leiten sollen, die man aber nie vollständig erreichen kann? Ich meine nein - eine ABWS ist unter der sich abzeichnenden Entwicklung in den Bereichen von Bildung und Wissenschaft zugunsten von offenen, freien, kollaborativen Produktions- und Nutzungsformen von Bildung und Wissenschaft eine realistische Alternative zu der Vielzahl der kleinteiligen, immer wieder eingeschränkten Schrankenregelungen.

\section{Auch das Wissenschafts- urheberrecht bleibt weiter Gegenstand eines beharrlichen Bohrens dicker Bretter}

Erneut ist es so gekommen, wie es das Struck'sche Gesetz prognostiziert, nämlich dass kein Gesetz den Bundestag so verlässt, wie es als Vorschlag des zuständigen Ministeriums hereingekommen ist. Was zu Beginn des Prozesses in den meisten Stellungnahmen (bis auf die aus der Verlagswelt) noch als Schritt in die richtige Richtung gewürdigt wurde, gilt am Ende wohl nur noch bedingt. $\mathrm{Zu}$ viel ist an verschiedenen Stellen wieder zurückgenommen worden (z. B. der Umfang der Nutzungserlaubnis von 25 auf 15 Prozent), und nichts von den vielen Veränderungs-/Verbesserungsvorschlägen (vgl. Fußnote 3), auch nicht die des Bundesrats (vgl. Fußnote 4), ist von der Bundesregierung ${ }^{39}$

39 Gegenäußerung der Bundesregierung zu der Stellungnahme des Bundesrates - http://dip21.bundestag.de/dip21/btd/18/123/1812378 .pdf. 
(und auch nicht von der Mehrheit des Bundestags) berücksichtigt worden.

Ein besonders krasses Beispiel ist das Verbot, Zeitungsartikel genehmigungsfrei (nicht vergütungsfrei) für Zwecke der Wissenschaft (Forschung und Lehre) zu nutzen - eine seit 2003 in $\S 52$ a des Urheberrechts bestehende und von Niemand bis dahin in Frage gestellte Erlaubnis. $\S 52 \mathrm{a}$ wird nicht mehr bestehen. Die $\S \S 60 \mathrm{a}, \mathrm{b}$, c, e und $\mathrm{f}$ untersagen die Nutzung von Zeitungsartikeln ab dem 1. März 2018.

Andere Regelungen sind nicht mehr zeitgemäß, z.B. dass man nicht von einem beliebigen Ort elektronisch (über VPN) auf digitalisierte Bestände der Bibliotheken zugreifen darf, sondern in die Räume der Bibliothekengehen muss. Wo Bibliotheken längst auch elektronische, virtuelle Räume sind. Diese Beschränkung sieht selbst die EU-Kommission nicht mehr als zeitgemäß an.

Entscheidend für eine insgesamt zurückhaltende Einschätzung ist vor allem die Entscheidung des Gesetzgebers, die Nutzungsintensität von publizierten (und urheberrechtsgeschützten) Werken jetzt zwar nicht mehr über unbestimmte Rechtsbegriffe wie „kleine Teile“, sondern quantitativ genau zu bestimmen - mal mit 15 Prozent, mal mit 10 Prozent und dann auch mit 75 Prozent.

Quantifizierungen schaffen sicher die gewünschte Rechtssicherheit, sind aber keineswegs zeitgemäß und erst recht nicht zukunftsoffen. Und das ist auch eine Ansage gegen die im Koalitionsvertrag von 2013 nicht bloß geforderte, sondern versprochene Allgemeine Bildungs- und Wissenschaftsschranke. Da hilft es auch nicht, dass die meisten Politiker aus der Regierungskoalition das jetzige Gesetz als Einlösen dieses Versprechens bezeichnen.

Eine Allgemeine Bildungs- und Wissenschaftsschranke muss Klauselcharakter haben: Der Umfang der Nutzung darf alleine durch den Zweck von Forschung und Lehre bestimmt werden. Wieso darf der Gesetzgeber in Wissenschaft derart eingreifen, dass er Forschern und Lehrenden/Lernenden vorschreibt, in welchem Umfang Werke genutzt werden dürfen?

$\mathrm{Zu}$ einer Allgemeinen Bildungs- und Wissenschaftsschranke hätte auch gehört, dass die Vergütungsfrage nicht nur mit dem „wie zu vergüten“ (ist jetzt im Gesetz richtigerweise mit der Pauschalierung beantwortet) beantwortet wird, sondern auch dass das grundsätzlichere „ob“ auf den Prüfstand gestellt würde. Angesichts der Tatsache, dass alle Urheber in Bildung und Wissenschaft zugleich immer schon Nutzer sind und damit alle Interesse haben, publiziertes Wissen so frei wie irgend möglich nutzen $\mathrm{zu}$ können, wird das Hindernis des Vergütungsanspruchs problematisch. Ohnehin ist es mehr als fraglich, ob für die in der Produktion mit öffentlichen Mitteln unterstützten und in öffentlichen Umgebungen produzierten Werken ein Vergütungsanspruch überhaupt noch besteht.

Dennoch, trotz mancher, durchaus auch grundlegender Kritik wird man nicht darum kommen anzuerkennen, dass das jetzige Gesetz in dem meisten Teilen ein Fortschritt gegenüber den dann wegfallenden Regelungen darstellt (vor allem in den $\S \S 52$ a und b, Teilen von 53 sowie $53 a)$.

Das Gesetz leistet zweifellos einen Beitrag zur Rechtssicherheit. Aber Rechtssicherheit ist kein Selbstzweck. Zweifel bestehen, ob das neue Gesetz schon jetzt noch zeitgemäß ist geschweige denn den zu erwartenden zukünftigen Entwicklungen gerecht werden kann. Ein Gesetz ist umso zukunftssicherer, je weniger es versucht, konkret zu definieren und zu regeln. Das jetzige UrhWissG bleibt regulierungstechnisch überspezifisch problematisch.

Auch das Urheberrecht ist Gegenstand eines langwierigen Bohrens dicker Bretter. Offensichtlich ist die Zeit für eine umfassendere Reform des Wissenschaftsurheberrechts noch nicht reif gewesen.

Es ist allerdings zu befürchten, dass die Politik das Wissenschaftsurheberrecht erst einmal für befriedigt einschätzt und kaum Anstalten machen wird, es in der nächsten Legislaturperiode noch einmal auf die Agenda zu setzen. Befördert wird das dadurch, dass nach vier Jahren eine Evaluierung angesetzt wird, auf Grund deren entschieden werden sollen, ob das jetzt beschlossene Gesetz nach einem weiteren Jahr auslaufen soll (was aber wohl kaum möglich ist, da wohl kaum dadurch die jetzt gelöschten Paragraphen wieder eingesetzt werden), weiterlaufen oder modifiziert werden soll. Ob durch eine Veränderung in der Regierungskoalition nach den Bundestagswahlen wieder Bewegung in das Wissenschaftsurheberrecht kommt (in welche Richtung auch immer), bleibt abzuwarten. Allerdings ist nicht auszuschließen, dass durch die Vorgabe einer (derzeit noch intensiv diskutierten) Urheberrechts-Richtlinie aus der $\mathrm{EU}^{40}$ dann ein Umsetzungszwang in den EU-Mitgliedsländern entsteht.

\section{Anhang - Text der $\S \S 60 a-60$}

\section{$7.1 \S 60$ a Unterricht und Lehre}

(1) Zur Veranschaulichung des Unterrichts und der Lehre an Bildungseinrichtungen dürfen zu nicht-kommerziellen

40 Vgl. Rainer Kuhlen: Zum Vorschlag der EU-Kommission für eine neue Urheberrechtsrichtlinie. Information - Wissenschaft \& Praxis (IWP) 2017; 68(2-3): 139-153. 
Zwecken bis zu 25 Prozent eines veröffentlichten Werkes vervielfältigt, verbreitet, öffentlich zugänglich gemacht und in sonstiger Weise öffentlich wiedergegeben werden.

1. für Lehrende und Teilnehmer der jeweiligen Veranstaltung,

2. für Lehrende und Prüfer an derselben Bildungseinrichtung sowie

3. für Dritte, soweit dies der Präsentation des Unterrichts, von Unterrichts- oder Lernergebnissen an der Bildungseinrichtung dient.

(2) Abbildungen, einzelne Beiträge aus derselben Fachzeitschrift oder wissenschaftlichen Zeitschrift, sonstige Werke geringen Umfangs und vergriffene Werke dürfen abweichend von Absatz 1 vollständig genutzt werden.

(3) Nicht durch die Absätze 1 und 2 erlaubt sind folgende Nutzungen:

1. Vervielfältigung durch Aufnahme auf Bild- oder Tonträger und öffentliche Wiedergabe eines Werkes, während es öffentlich vorgetragen, aufgeführt oder vorgeführt wird,.

2. Vervielfältigung, Verbreitung und öffentliche Wiedergabe eines Werkes, das ausschließlich für den Unterricht an Schulen geeignet, bestimmt und entsprechend gekennzeichnet ist, an Schulen sowie.

3. Vervielfältigung von grafischen Aufzeichnungen von Werken der Musik.

(4) Bildungseinrichtungen sind frühkindliche Bildungseinrichtungen, Schulen, Hochschulen sowie Einrichtungen der Berufsbildung oder der sonstigen Aus- und Weiterbildung.

\section{$7.2 \S 60 \mathrm{~b}$ Unterrichts- und Lehrmedien}

(1) Hersteller von Unterrichts- und Lehrmedien dürfen für solche Sammlungen bis zu 10 Prozent eines veröffentlichten Werkes vervielfältigen, verbreiten und öffentlich zugänglich machen.

\section{(2) § 60a Absatz 2 und 3 ist entsprechend anzuwenden.}

(3) Unterrichts- und Lehrmedien im Sinne dieses Gesetzes sind Sammlungen, die Werke einer größeren Anzahl von Urhebern vereinigen und ausschließlich zur Veranschaulichung des Unterrichts und der Lehre an Bildungseinrichtungen ( $\$ 60 \mathrm{a}$ ) zu nicht-kommerziellen Zwecken geeignet, bestimmt und entsprechend gekennzeichnet sind.

\section{$7.3 \S 60$ c Wissenschaftliche Forschung}

(1) Zum Zweck der nicht-kommerziellen wissenschaftlichen Forschung dürfen bis zu 25 Prozent eines Werkes vervielfältigt, verbreitet und öffentlich zugänglich gemacht werden

1. für einen bestimmt abgegrenzten Kreis von Personen für deren eigene wissenschaftliche Forschung sowie

2. für einzelne Dritte, soweit dies der Überprüfung der Qualität wissenschaftlicher Forschung dient.

(2) Für die eigene wissenschaftliche Forschung dürfen bis zu 75 Prozent eines Werkes vervielfältigt werden.

(3) Abbildungen, einzelne Beiträge aus derselben Fachzeitschrift oder wissenschaftlichen Zeitschrift, sonstige Werke geringen Umfangs und vergriffene Werke dürfen abweichend von den Absätzen 1 und 2 vollständig genutzt werden.

(4) Nicht durch die Absätze 1 bis 3 erlaubt ist es, während öffentlicher Vorträge, Aufführungen oder Vorführungen eines Werkes diese auf Bild- oder Tonträger aufzunehmen und später öffentlich zugänglich zu machen.

\section{$7.4 \S 60 \mathrm{~d}$ Text und Data Mining}

(1) Um eine Vielzahl von Werken (Ursprungsmaterial) für die wissenschaftliche Forschung automatisiert auszuwerten, ist es zulässig,

1. das Ursprungsmaterial auch automatisiert und systematisch zu vervielfältigen, um daraus insbesondere durch Normalisierung, Strukturierung und Kategorisierung ein auszuwertendes Korpus zu erstellen, und

2. das Korpus einem bestimmt abgegrenzten Kreis von Personen für die gemeinsame wissenschaftliche Forschung sowie einzelnen Dritten zur Überprüfung der Qualität wissenschaftlicher Forschung öffentlich zugänglich zu machen.

Der Nutzer darf hierbei nur nicht-kommerzielle Zwecke verfolgen.

(2) Werden Datenbankwerke nach Maßgabe des Absatzes 1 genutzt, so gilt dies als übliche Benutzung nach $\S 55 \mathrm{a}$ Satz 1. Werden unwesentliche Teile von Datenbanken nach Maßgabe des Absatzes 1 genutzt, so gilt dies mit der normalen Auswertung der Datenbank sowie mit den berechtigten Interessen des Datenbankherstellers im Sinne von $\S 87 \mathrm{~b}$ Absatz 1 Satz 2 und $\S 87 \mathrm{e}$ als vereinbar. 
(3) Das Korpus und die Vervielfältigungen des Ursprungsmaterials sind nach Abschluss der Forschungsarbeiten zu löschen; die öffentliche Zugänglichmachung ist zu beenden. Zulässig ist es jedoch, das Korpus und Vervielfältigungen des Ursprungsmaterials den in den $\S \S 60$ e und $60 \mathrm{f}$ genannten Institutionen zur dauerhaften Aufbewahrung zu übermitteln.

\section{$7.5 \S 60$ e Bibliotheken}

(1) Öffentlich zugängliche Bibliotheken, die keine unmittelbaren oder mittelbaren kommerziellen Zwecke verfolgen (Bibliotheken), dürfen ein Werk aus ihrem Bestand oder ihrer Ausstellung für Zwecke der Zugänglichmachung, Indexierung, Katalogisierung, Erhaltung und Restaurierung vervielfältigen oder vervielfältigen lassen, auch mehrfach und mit technisch bedingten Änderungen.

(2) Verbreiten dürfen Bibliotheken Vervielfältigungen eines Werkes aus ihrem Bestand an andere Bibliotheken oder an in $\S 60 \mathrm{f}$ genannte Institutionen für Zwecke der Restaurierung. Verleihen dürfen sie restaurierte Werke sowie Vervielfältigungsstücke von Zeitungen, vergriffenen oder zerstörten Werken aus ihrem Bestand.

(3) Verbreiten dürfen Bibliotheken Vervielfältigungen eines in $\S 2$ Absatz 1 Nummer 4 bis 7 genannten Werkes, sofern dies in Zusammenhang mit dessen öffentlicher Ausstellung oder zur Dokumentation des Bestandes der Bibliothek erfolgt.

(4) Zugänglich machen dürfen Bibliotheken an Terminals in ihren Räumen ein Werk aus ihrem Bestand ihren Nutzern für deren Forschung oder private Studien. Sie dürfen den Nutzern je Sitzung Vervielfältigungen an den Terminals von bis zu 10 Prozent eines Werkes sowie von einzelnen Abbildungen, Beiträgen aus derselben Fachzeitschrift oder wissenschaftlichen Zeitschrift, sonstigen Werken geringen Umfangs und vergriffenen Werken zu nicht-kommerziellen Zwecken ermöglichen.

(5) Auf Einzelbestellung an Nutzer zu nicht-kommerziellen Zwecken übermitteln dürfen Bibliotheken Vervielfältigungen von bis zu 10 Prozent eines erschienenen Werkes sowie einzelne Beiträge, die in Fachzeitschriften oder wissenschaftlichen Zeitschriften erschienen sind.

\section{$7.6 \S 60 \mathrm{f}$ Archive, Museen und Bildungseinrichtungen}

(1) Für Archive, Einrichtungen im Bereich des Film- oder Tonerbes sowie öffentlich zugängliche Museen und Bildungseinrichtungen ( $\$ 60$ a Absatz 4), die keine unmittelbaren oder mittelbaren kommerziellen Zwecke verfolgen, gilt $\S 60$ e mit Ausnahme des Absatzes 5 entsprechend.

(2) Archive, die auch im öffentlichen Interesse tätig sind, dürfen ein Werk vervielfältigen oder vervielfältigen lassen, um es als Archivgut in ihre Bestände aufzunehmen, wenn die abgebende Stelle unverzüglich die bei ihr vorhandenen Vervielfältigungen löscht.

\section{$7.7 \S 60 \mathrm{~g}$ Gesetzlich erlaubte Nutzung und vertragliche Nutzungsbefugnis}

(1) Soweit eine Einräumung oder Versagung einer Nutzungsbefugnis eine nach diesem Unterabschnitt erlaubte Nutzung betrifft, ist die Vereinbarung unwirksam.

(2) Eine Vereinbarung über die Zugänglichmachung an Terminals nach $\S 60$ e Absatz 4 und $\S 60$ f Absatz 1 geht abweichend von Absatz 1 der gesetzlichen Erlaubnis vor.

\section{$7.8 \S 60 \mathrm{~h}$ Angemessene Vergütung der gesetzlich erlaubten Nutzungen}

(1) Für Nutzungen nach Maßgabe dieses Unterabschnitts hat der Urheber Anspruch auf Zahlung einer angemessenen Vergütung. Vervielfältigungen sind nach den $\S \S 54$ bis $54 \mathrm{c}$ zu vergüten.

(2) Folgende Nutzungen sind abweichend von Absatz 1 vergütungsfrei:

1. die öffentliche Wiedergabe für Angehörige von Bildungseinrichtungen und deren Familien nach $\S 60 \mathrm{a}$ Absatz 1 Nummer 1 und 3 sowie Absatz 2 mit Ausnahme der öffentlichen Zugänglichmachung,

2. Vervielfältigungen zum Zweck der Indexierung, Katalogisierung, Erhaltung und Restaurierung nach $\S 60 \mathrm{e}$ Absatz 1 und $\S 60 \mathrm{f}$ Absatz 1.

(3) Eine pauschale Vergütung oder eine repräsentative Stichprobe der Nutzung für die nutzungsabhängige Berechnung der angemessenen Vergütung genügt. Dies gilt nicht bei Nutzungen nach den $\S \S 60 \mathrm{~b}$ und 60 e Absatz 5. 
(4) Der Anspruch auf angemessene Vergütung kann nur durch eine Verwertungsgesellschaft geltend gemacht werden.

(5) Ist der Nutzer im Rahmen einer Einrichtung tätig, so ist nur sie die Vergütungsschuldnerin. Für Vervielfältigungen, die gemäß Absatz 1 Satz 2 nach den $\S \S 54$ bis 54c abgegolten werden, sind nur diese Regelungen anzuwenden.

\section{8 Änderung des Gesetzes über die Deutsche Nationalbibliothek}

\section{§ 16 a Urheberrechtlich erlaubte Nutzungen}

(1) Die Bibliothek darf Medienwerke in unkörperlicher Form für eigene und fremde Pflichtexemplarbestände vergütungsfrei vervielfältigen und übermitteln, auch automatisiert und systematisch. Dies gilt nur, soweit die Medienwerke entweder ohne Beschränkungen, insbesondere für jedermann und unentgeltlich, öffentlich zugänglich oder zur Abholung durch die Bibliothek bereitgestellt sind. Die nach den Sätzen 1 und 2 erstellten Vervielfältigungen dürfen anschließend wie andere Bestandswerke weitergenutzt werden.

(2) Die Bibliothek darf im Auftrag eines Nutzers Werke oder andere nach dem Urheberrechtsgesetz geschützte Schutzgegenstände für die nicht kommerzielle wissenschaftliche Forschung zur Erleichterung von Zitaten vergütungsfrei vervielfältigen und unter einer dauerhaft gleichbleibenden Internetadresse öffentlich zugänglich machen. Dies gilt nur, wenn die Werke und sonstigen Schutzgegenstände ohne Beschränkungen, insbesondere für jedermann und unentgeltlich, öffentlich zugänglich sind und zudem ihre dauerhafte Erreichbarkeit nicht durch die Bibliothek selbst oder durch Dritte gesichert ist, etwa dadurch, dass die Werke und sonstigen Schutzgegenstände über andere, entgeltliche oder unentgeltliche Dienste erreichbar sind.

\section{Abkürzungen}

ABWK - Allgemeine Bildungs- und Wissenschaftsklausel ABWS - Allgemeine Bildungs- und Wissenschaftsschranke BMJV - Bundesministerium der Justiz und für Verbraucherschutz

BGH - Bundesgerichtshof

DINI - Deutsche Initiative für Netzwerkinformation e.V.

EuGH - Europäischer Gerichtshof

$\mathrm{Hlb}$ - Hochschullehrerbund

IDS - Institut für Deutsche Sprache

RefE - Referentenentwurf

RegE - Regierungsentwurf

VDV - Verband Deutscher Bühnen- und Medienverlage

VDZ - Verband Deutscher Zeitschriftenverleger

WIPO - Weltorganisation für geistiges Eigentum (World Intellectual Property Organization)

ZUM - Zeitschrift für Urheber- und Medienrecht

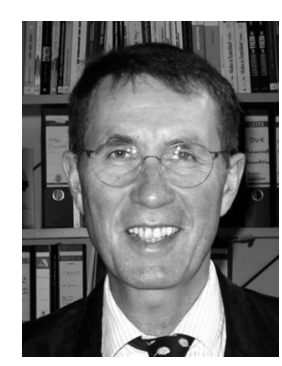

Prof. em. Dr. Rainer Kuhlen

Bogotastraße 4

14163 Berlin

www.kuhlen.name

http://orcid.org/0000-0002-4497-6422

rainer.kuhlen@uni-konstanz.de

Dr. Rainer Kuhlen ist emeritierter Professor für Informationswissenschaft im Fachbereich Informatik und Informationswissenschaft an der Universität Konstanz und langjähriger Sprecher des Aktionsbündnisses „Urheberrecht für Bildung und Wissenschaft“. 